\title{
The seismic properties of low-mass He-core white dwarf stars ${ }^{\star}$
}

\author{
A. H. Córsico ${ }^{1,2}$, A. D. Romero ${ }^{1,2}$, L. G. Althaus ${ }^{1,2}$, and J. J. Hermes ${ }^{3,4}$ \\ ${ }^{1}$ Facultad de Ciencias Astronómicas y Geofísicas, Universidad Nacional de La Plata, Paseo del Bosque s/n, 1900 La Plata, Argentina \\ 2 CCT La Plata, CONICET, 1900 La Plata, Argentina \\ 3 Department of Astronomy, University of Texas at Austin, Austin, TX 78712, USA \\ ${ }^{4}$ McDonald Observatory, Fort Davis, TX 79734, USA \\ e-mail: [acorsico; aromero; althaus]@fcaglp.unlp.edu.ar; jjhermes@astro.as.utexas.edu
}

Received 27 July 2012 / Accepted 25 September 2012

\begin{abstract}
Context. In recent years, many low-mass $\left(\lessgtr 0.45 M_{\odot}\right)$ white dwarf stars expected to harbor He cores have been detected in the field of the Milky Way and in several galactic globular and open clusters. Until recently, no objects of this kind showed pulsations. This situation has changed recently with the exciting discovery of SDSS J184037.78+642312.3, the first pulsating low-mass white dwarf star.

Aims. Motivated by this extremely important finding, and in view of the very valuable asteroseismological potential of these objects, we present here a detailed pulsational study applied to low-mass He-core white dwarfs with masses ranging from 0.17 to $0.46 M_{\odot}$, based on full evolutionary models representative of these objects. This study is aimed to provide a theoretical basis from which to interpret future observations of variable low-mass white dwarfs.

Methods. The background stellar models on which our pulsational analysis was carried out were derived by taking into account the complete evolutionary history of the progenitor stars, with special emphasis on the diffusion processes acting during the white dwarf cooling phase. We computed nonradial $g$-modes to assess the dependence of the pulsational properties of these objects with stellar parameters such as the stellar mass and the effective temperature, and also with element diffusion processes. We also performed a $g$ - and $p$-mode pulsational stability analysis on our models and found well-defined blue edges of the instability domain, where these stars should start to exhibit pulsations.

Results. We found substantial differences in the seismic properties of white dwarfs with $M_{*} \gtrsim 0.20 M_{\odot}$ and the extremely low-mass (ELM) white dwarfs $\left(M_{*} \lesssim 0.20 M_{\odot}\right)$. Specifically, $g$-mode pulsation modes in ELM white dwarfs mainly probe the core regions and are not dramatically affected by mode-trapping effects by the $\mathrm{He} / \mathrm{H}$ interface, whereas the opposite is true for more massive He-core white dwarfs. We found that element diffusion processes substantially affects the shape of the $\mathrm{He} / \mathrm{H}$ chemical transition region, leading to non-negligible changes in the period spectrum of low-mass white dwarfs, in particular in the range of stellar masses characteristic of ELM objects. Finally, our stability analysis successfully predicts the pulsations of the only known variable low-mass white dwarf (SDSS J184037.78+642312.3) at the right effective temperature, stellar mass and range of periods.

Conclusions. Our computations predict both $g$ - and $p$-mode pulsational instabilities in a significant number of known low-mass and ELM white dwarfs. It is worth observing these stars in order to discover if they pulsate.
\end{abstract}

Key words. asteroseismology - stars: oscillations - white dwarfs - stars: evolution - stars: interiors

\section{Introduction}

White dwarf stars are the most common evolutionary fate of low- and intermediate-mass $\left(M_{*} \lesssim 11 M_{\odot}\right.$; Siess 2007) stars. Indeed, the vast majority - more than $95 \%$ - of all stars will die as white dwarfs. As such, these old and compact stellar remnants provide a wealth of information about the evolution of stars, star formation, and the age of a variety of stellar populations, such as our Galaxy and open and globular clusters (Winget \& Kepler 2008; García-Berro et al. 2010; Althaus et al. 2010). Among the different flavors of white dwarfs, the most common is the spectral class of DA white dwarfs, characterized by $\mathrm{H}$ rich envelopes, that comprises around $80 \%$ of all known white dwarfs. An important property of the white dwarf population is their mass distribution. For DA white dwarfs, the mass distribution peaks at $\approx 0.59 M_{\odot}$, and exhibits also high-mass and

* Stellar models and tabulated pulsation periods are available at the CDS via anonymous ftp to

cdsarc.u-strasbg.fr $(130.79 .128 .5)$ or via

http://cdsarc.u-strasbg.fr/viz-bin/qcat?J/A+A/547/A96 low-mass components (Kepler et al. 2007; Tremblay et al. 2011; Kleinman et al. 2012). The population of low-mass white dwarfs has masses lower than $0.45 M_{\odot}$ and peaks at $\approx 0.39 M_{\odot}$. Recently, a large number of low-mass white dwarfs with masses below 0.20-0.25 $M_{\odot}$ has been discovered (Kawka \& Vennes 2009; Brown et al. 2010, 2012; Kilic et al. 2011, 2012); they are referred to as extremely low-mass (ELM) white dwarfs.

The low-mass white dwarf population is probably produced by strong mass-loss episodes at the red giant branch (RGB) phase before the He-flash onset. As such, these white dwarfs are expected to harbor He cores, in contrast to average mass white dwarfs, which all likely contain $\mathrm{C} / \mathrm{O}$ cores. For solar metallicity progenitors $(Z \approx 0.01-0.02)$, mass-loss episodes must occur in binary systems through mass-transfer, since single star evolution is not able to predict the formation of these stars in a Hubble time. This evolutionary scenario is confirmed by the fact that most of low-mass white dwarfs are found in binary systems (e.g., Marsh et al. 1995), and usually as companions to millisecond pulsars (van Kerkwijk et al. 2005). In particular, binary evolution is the most likely origin for ELM white dwarfs 
(Marsh et al. 1995). On the other hand, for high-metallicity progenitors $(Z \approx 0.03-0.05)$, the He-core flash can probably be avoided by mass losses due to strong stellar winds on the RGB. This can be the origin of the isolated low-mass He-core white dwarfs $\left(M_{*} \approx 0.4 M_{\odot}\right)$ found in the high-metallicity open cluster NGC 6791 (Hansen 2005; Kalirai et al. 2007; García-Berro et al. 2010).

The evolution of low-mass white dwarfs is strongly dependent on their stellar mass and the occurrence of element diffusion processes. Althaus et al. (2001) and Panei et al. (2007) have demonstrated that element diffusion leads to a dichotomy regarding the thickness of the $\mathrm{H}$ envelope, which translates into a dichotomy in the age of low-mass He-core white dwarfs. Specifically, for stars with $M_{*} \gtrsim 0.18-0.20 M_{\odot}$, the white dwarf progenitor experiences multiple diffusion-induced thermonuclear flashes that burn most of the $\mathrm{H}$ content of the envelope, and as a result, the remnant enters the final cooling track with a very thin $\mathrm{H}$ envelope. In this way, the star is unable to sustain stable nuclear burning while cools and the evolutionary timescale is rather short $\left(\approx 10^{7} \mathrm{yr}\right)^{1}$. On the other hand, if $M_{*} \lesssim 0.20 M_{\odot}$, the white dwarf progenitor does not experience $\mathrm{H}$ flashes at all, and the remnant enters its terminal cooling branch with a thick $\mathrm{H}$ envelope. This is thick enough for residual $\mathrm{H}$ nuclear burning to become the main energy source, that ultimately slows down the evolution, in which case the cooling timescale is of the order $\approx 10^{9}$ yrs. The age dichotomy has been also suggested by observations of those low-mass He-core white dwarfs that are companions to millisecond pulsars (Bassa et al. 2003; Bassa 2006).

The internal structure of white dwarfs can be disentangled by means of asteroseismology (Winget \& Kepler 2008; Fontaine \& Brassard 2008; Althaus et al. 2010). White dwarf asteroseismology allows us to place constraints on the stellar mass, the thickness of the compositional layers, and the core chemical composition, among other relevant properties. In connection with the core composition of white dwarfs, some theoretical work in the past has explored the differences that should be expected in the pulsation properties of low-mass white dwarfs harboring cores made either of $\mathrm{C} / \mathrm{O}$ or He (Althaus et al. 2004), and high mass white dwarfs with cores made either of $\mathrm{C} / \mathrm{O}$ or O/Ne (Córsico et al. 2004). More recently, Castanheira \& Kepler (2008, 2009), and Romero et al. (2012) (see also Romero 2012) have carried out detailed asteroseismological studies on a large number of pulsating DA white dwarfs (DAV or ZZ Ceti variables), providing valuable information about the range of the $\mathrm{H}$ envelope thicknesses expected for the class of DA white dwarfs.

In order to apply the principles of asteroseismology on low-mass and ELM white dwarfs, it is necessary to find such stars (either in the Galactic field or in stellar clusters) undergoing pulsations, something that until very recently had not been possible. In this regard, it is worth mentioning that exhaustive searches reported by Steinfadt et al. (2012) have given null results, despite their theoretical predictions (Steinfadt et al. 2010, hereinafter SEA10) suggesting pulsational instabilities at least in stars with $M_{*} \lesssim 0.20 M_{\odot}$ (ELM white dwarfs). The situation has improved drastically with the exciting discovery of SDSS J184037.78+642312.3, the first pulsating ELM white

\footnotetext{
1 Note that, if element diffusion processes are not included, theoretical computations also predict the occurrence of $\mathrm{H}$-shell flashes before the terminal cooling branch is reached, but the $\mathrm{H}$ envelopes remain thick, with substantial $\mathrm{H}$ burning and large white dwarf cooling ages (Driebe et al. 1998; Sarna et al. 2000).
}

dwarf (Hermes et al. 2012). This star $\left(T_{\text {eff }}=9100 \pm 170 \mathrm{~K}\right.$, $\log g=6.22 \pm 0.06)$ exhibits multiperiodic photometric variations with a dominant period at $\approx 4698 \mathrm{~s}$, much longer than the longest periodicities detected in any ZZ Ceti star (100-1200 s). The discovery of this pulsating ELM white dwarf opens the possibility of sounding the interiors of low-mass white dwarfs employing the tools of asteroseismology.

Needless to say, accurate and realistic stellar models representative of low-mass white dwarfs are needed to correctly interpret the present and future observations of pulsations in these objects. In particular, the complete evolutionary history of the progenitor stars must be taken fully into account in order to assess the correct thermo-mechanical structure of the white dwarf and the thickness of the $\mathrm{H}$ envelope remaining after multiple diffusion-induced CNO flashes occurring before the terminal cooling branch is reached, which strongly determines the cooling timescales. Also, element diffusion processes must be considered in order to consistently account for the evolving shape of the internal chemical profiles (and, in particular, the chemical transition regions) during the white dwarf cooling phase. Finally, stable $\mathrm{H}$ burning, which is particularly relevant in the case of ELM white dwarfs (and can play an important role in the excitation of pulsations), must be taken into account as well.

Motivated by the asteroseismic potential of pulsating lowmass white dwarfs, and stimulated by the discovery of the first variable ELM white dwarf, we have started in the La Plata Observatory a theoretical study of the pulsation properties of low-mass, He-core white dwarfs with masses in the range $0.17-0.46 M_{\odot}$. Our interest in these stars is strengthened by the availability of detailed and fully evolutionary stellar models extracted from the sequences of low-mass He-core white dwarfs presented by Althaus et al. (2009). These models were derived by considering the evolutionary history of progenitor stars with high metallicity, and taking into account a self-consistent, timedependent treatment of the gravitational settling and chemical diffusion, as well as of residual nuclear burning. In this study, we explore the adiabatic pulsation properties of these models, that is, the expected range of periods and period spacings, the propagation characteristics of the pulsation modes, the regions of period formation, as well as the dependence on the effective temperature and stellar mass. For the first time, we assess the pulsation properties of He-core white dwarfs with masses in the range $0.20-0.45 M_{\odot}$. In particular, we highlight the expected differences in the seismic properties of objects with $M_{*} \gtrsim 0.20 M_{\odot}$ and the ELM white dwarfs $\left(M_{*} \lesssim 0.20 M_{\odot}\right)$. Since the most important work in the literature on pulsations of low-mass white dwarfs is the study of SEA10, we shall invoke it repeatedly to compare their results with our own findings, in particular regarding the seismic properties of ELM white dwarfs. We also explore the role of time-dependent element diffusion in ELM models on their pulsational properties, an aspect not discussed by SEA10. In addition, we compute $g$ - and $p$-mode blue edges of the instability domain of these stars through a nonadiabatic stability analysis of our sequences, and in particular, we try to determine whether our computations are able to predict the pulsations exhibited by SDSS J184037.78+642312.3. This paper is organized as follows. In Sect. 2 we briefly describe our numerical tools and the main ingredients of the evolutionary sequences we use to assess the pulsation properties of low-mass He-core white dwarfs. In Sect. 3 we present in detail our adiabatic pulsation results, while in Sect. 4 we describe our nonadiabatic results and the case of SDSS J184037.78+642312.3. Finally, in Sect. 5 we summarize the main findings of the paper. 


\section{Computational tools and evolutionary sequences}

\subsection{Evolutionary code and input physics}

The evolutionary models employed in our pulsational analysis were generated with the LPCODE evolutionary code, which produces complete and detailed white dwarfs models incorporating very updated physical ingredients. While detailed information about LPCODE can be found in Althaus et al. (2005, 2009) and references therein, we highlight here only those ingredients which are important for our analysis of low-mass, He-core white dwarf stars.

(a) The standard mixing length theory (MLT) for convection (with the free parameter $\alpha=1.6$ ) has been adopted. Had we adopted a different convective efficiency, then the adiabatic pulsation results presented in this work (Sect. 3) should not be affected, although the predicted blue edge of the ELM and low-mass He-core white dwarfs instability domain (Sect. 4) should appreciably change.

(b) A supersolar metallicity for the progenitor stars has been considered: $Z=0.03$.

(c) Radiative opacities for arbitrary metallicity in the range from 0 to 0.1 are from the OPAL project (Iglesias \& Rogers 1996) supplemented at low temperatures with the molecular opacities of Alexander \& Ferguson (1994).

(d) Neutrino emission rates for pair, photo, and bremsstrahlung processes were taken from Itoh et al. (1996), and for plasma processes we included the treatment of Haft et al. (1994).

(e) Conductive opacities are those of Cassisi et al. (2007).

(f) For the white dwarf regime we employed an updated version of the Magni \& Mazzitelli (1979) equation of state.

(g) The nuclear network takes into account 16 elements and 34 nuclear reactions for pp chains, CNO bi-cycle, He burning, and $\mathrm{C}$ ignition.

(h) Time-dependent diffusion due to gravitational settling and chemical and thermal diffusion of nuclear species was taken into account following the multicomponent gas treatment of Burgers (1969).

(i) Abundance changes were computed according to element diffusion, nuclear reactions, and convective mixing. This detailed treatment of abundance changes by different processes during the white dwarf regime constitutes a key aspect in the evaluation of the importance of residual nuclear burning for the cooling of low-mass white dwarfs.

\subsection{Pulsation codes}

In this work, we have carried out a detailed adiabatic $g$-mode pulsation study aimed at exploring the seismic properties of low-mass He-core white dwarf models. In addition, we have performed a $g$ - and $p$-mode stability analysis of our model sequences. The pulsation computations of our adiabatic survey were performed with the pulsation code described in detail in Córsico \& Althaus (2006), which is coupled to the LPCODE evolutionary code. The pulsation code is based on a general Newton-Raphson technique that solves the full fourth-order set of equations and boundary conditions governing linear, adiabatic, nonradial stellar pulsations following the dimensionless formulation of Dziembowski (1971) (see Unno et al. 1989). The prescription we follow to assess the run of the Brunt-Väisälä frequency $(N)$ for a degenerate environment typical of the deep interior of a white dwarf is the so-called "Ledoux modified" treatment (Tassoul et al. 1990).
As for the stability analysis, we have employed the finitedifference nonadiabatic pulsation code described in detail in Córsico et al. (2006). The code solves the full sixth-order complex system of linearized equations and boundary conditions as given by Unno et al. (1989). Typically, our adiabatic and nonadiabatic periods differ by less than $\approx 2 \%$. The caveat of our analysis is that the nonadiabatic computations rely on the frozenconvection approximation, in which the perturbation of the convective flux is neglected. While this approximation is known to give unrealistic locations of the $g$-mode red edge of instability, it leads to satisfactory predictions for the location of the blue edge of the ZZ Ceti (DAV) instability strip (see, e.g., Brassard $\&$ Fontaine 1999 and van Grootel et al. 2012) and also for the V777 Her (DBV) instability strip (see, for instance, Beauchamp et al. 1999 and Córsico et al. 2009).

\subsection{Evolutionary sequences}

To derive starting configurations for the He-core cooling sequences consistent with the evolutionary history of the progenitor star, Althaus et al. (2009) simply removed mass from a $1 M_{\odot}$ model at the appropriate stages of its evolution (Iben \& Tutukov 1986; Driebe et al. 1998). Other details about the procedure to obtain the initial models are provided in Althaus et al. (2009). The resulting final stellar masses $\left(M_{*} / M_{\odot}\right)$ are listed in Table 1 with the total amount of $\mathrm{H}$ contained in the envelope $\left(M_{\mathrm{H}} / M_{*}\right)$, and the coordinate $-\log (q)$ (being $q=1-M_{r} / M_{*}$ ) of the location of the $\mathrm{He} / \mathrm{H}$ chemical interface (where $X_{\mathrm{He}}=X_{\mathrm{H}}=0.5$ ), for models at $T_{\text {eff }} \approx 10000 \mathrm{~K}$. The last column shows the time spent by the stars to cool from $T_{\text {eff }} \approx 14000 \mathrm{~K}$ to $\approx 8000 \mathrm{~K}$.

We mention again that a metallicity of $Z=0.03$ for the progenitor stars has been adopted. This value is appropriate for overmetallic environments such as the open cluster NGC 6791 (Althaus et al. 2009). Had we adopted a solar metallicity value ( $Z \approx 0.01-0.02$ ) instead, then all our sequences would be characterized by $\mathrm{H}$ envelopes thicker than those considered here (second column of Table 1), but it would not qualitatively affect the results presented in this paper.

The sequence of $M_{*}=0.17 M_{\odot}$ does not correspond to the set of sequences presented by Althaus et al. (2009); it was specifically computed for the present study. The models of this sequence are representative of ELM white dwarfs. The evolutionary history of this sequence is quite different as compared with the remaining sequences, because its progenitor star does not experience any $\mathrm{CNO}$ flash. As a result, the remaining $\mathrm{H}$ envelope is markedly thicker than for the other sequences. The very distinct origin of the $0.17 M_{\odot}$ sequence is also emphatically evidenced by the time the star spends to cool from $\approx 14000$ to $\approx 8000 \mathrm{~K}$, which is about 3.7 times longer than for the case of the $0.198 M_{\odot}$ sequence.

The long time that ELM white dwarfs should spend at these effective temperatures, by virtue of vigorous stable $\mathrm{H}$ burning via the pp chain, motivated Steinfadt et al. (2012) to search for pulsating objects with masses lower than $\approx 0.20 M_{\odot}$. We note from Table 1 , however, that models with $\approx 0.40-0.45 M_{\odot}$ should also have comparable evolutionary timescales. This implies that there should be a good chance of finding pulsating objects with these masses, if they exist, and current searches of variable lowmass white dwarfs should not be restricted only to ELM white dwarfs.

In Fig. 1 we depict our evolutionary tracks on the plane $T_{\text {eff }}-\log g$ (black thin lines). In order to have an overview of the observational status, we have included the location of the low-mass white dwarfs known to date. They include field stars 


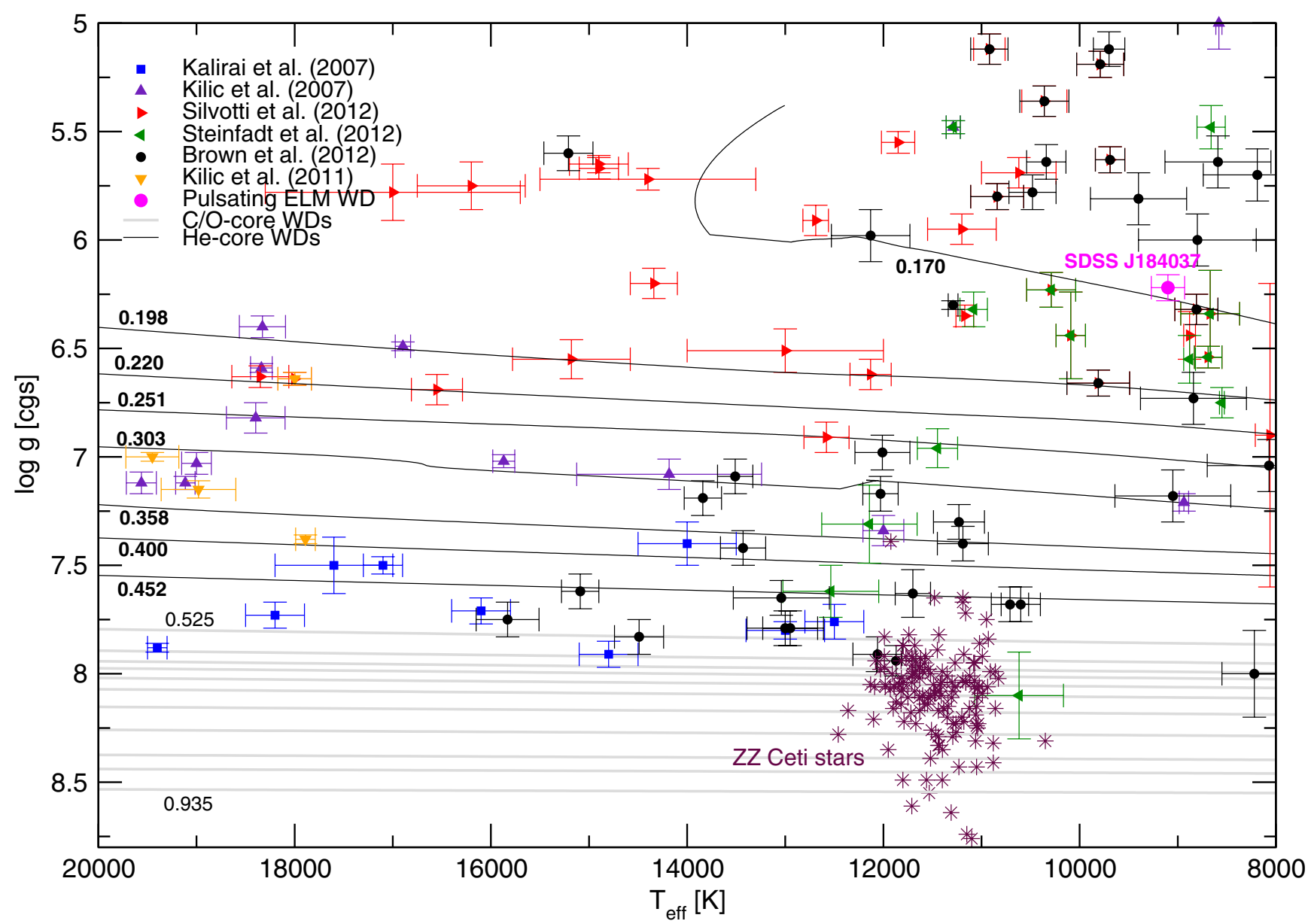

Fig. 1. The $T_{\text {eff }}-\log g$ diagram showing our low-mass He-core white dwarf evolutionary tracks (black thin lines). Bold numbers correspond to the stellar mass of each sequence. The different symbols with error bars represent the location of the low-mass white dwarfs known to date. We include field objects as well as stars found in the open cluster NGC 6791 to have a general overview of the observational status of these stars. The location of the first known pulsating ELM white dwarf $\left(T_{\text {eff }}=9100 \pm 170 \mathrm{~K}, \log g=6.22 \pm 0.06\right.$; Hermes et al. 2012) is displayed with a small circle (magenta). As reference, we also depict the C/O-core white dwarf evolutionary tracks (gray thick lines) for masses between 0.525 and $0.935 M_{\odot}$ (Romero et al. 2012) and the location of the known ZZ Ceti (DAV) stars.

Table 1. Selected properties of our He-core white dwarf sequences (metallicity of the progenitor star: $Z=0.03$ ) at $T_{\text {eff }} \approx 10000 \mathrm{~K}$.

\begin{tabular}{cccc}
\hline \hline$M_{*} / M_{\odot}$ & $M_{\mathrm{H}} / M_{*}\left[10^{-3}\right]$ & $-\log \left(1-M_{r} / M_{*}\right)$ & $\tau\left[\mathrm{Myr}=10^{6} \mathrm{yr}\right]$ \\
\hline 0.170 & 12.992 & 2.0196 & 2122.394 \\
0.198 & 3.7717 & 3.2851 & 576.575 \\
0.220 & 1.6621 & 3.8237 & 387.775 \\
0.251 & 1.2039 & 3.1733 & 580.847 \\
0.303 & 1.2661 & 3.0191 & 798.432 \\
0.358 & 0.5428 & 3.3759 & 917.173 \\
0.400 & 0.6638 & 3.2590 & 1204.545 \\
0.452 & 0.3708 & 3.5459 & 1228.023 \\
\hline
\end{tabular}

Notes. The table lists the stellar mass, the mass of $\mathrm{H}$ in the outer envelope, the location of the $\mathrm{He} / \mathrm{H}$ chemical interface, and the time it takes the star to cool from $T_{\text {eff }} \approx 14000 \mathrm{~K}$ to $\approx 8000 \mathrm{~K}$.

that are members of binary systems (Kilic et al. 2007, 2011; Brown et al. 2012; Steinfadt et al. 2012; Silvotti et al. 2012) and single stars residing in the open cluster NGC 6791 (Kalirai et al. 2007). As reference, we include also the location of the known ZZ Ceti stars and the evolutionary tracks corresponding to $\mathrm{C} / \mathrm{O}$-core white dwarfs (gray thick lines).

\section{Adiabatic pulsation properties}

\subsection{Asymptotic period spacing}

For $g$-modes with high radial order $k$ (long periods), the separation of consecutive periods $(|\Delta k|=1)$ becomes nearly constant at a value given by the asymptotic theory of nonradial stellar pulsations. Specifically, the asymptotic period spacing (Tassoul et al. 1990) is given by:

$\Delta \Pi_{\ell}^{\mathrm{a}}=\Pi_{0} / \sqrt{\ell(\ell+1)}$,

where

$\Pi_{0}=2 \pi^{2}\left[\int_{r_{1}}^{r_{2}} \frac{N}{r} \mathrm{~d} r\right]^{-1}$.

The squared Brunt-Väisälä frequency $(N)$ is computed as:

$N^{2}=\frac{g^{2} \rho}{P} \frac{\chi_{\mathrm{T}}}{\chi_{\rho}}\left[\nabla_{\mathrm{ad}}-\nabla+B\right]$,

where the compressibilities are defined as

$\chi_{\rho}=\left(\frac{\mathrm{d} \ln P}{\operatorname{dln} \rho}\right)_{\mathrm{T},\left\{\mathrm{X}_{\mathrm{i}}\right\}} \quad \chi_{\mathrm{T}}=\left(\frac{\mathrm{d} \ln P}{\mathrm{~d} \ln T}\right)_{\rho,\left\{\mathrm{X}_{\mathrm{i}}\right\}}$. 


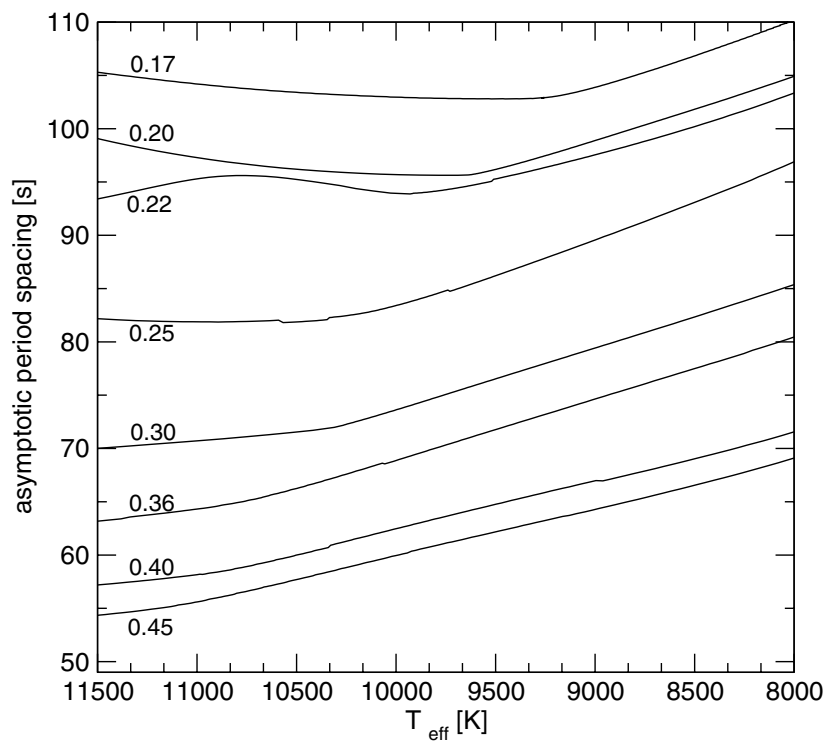

Fig. 2. The asymptotic period spacing in terms of the effective temperature for all of our low-mass He-core evolutionary sequences.

The Ledoux term $B$ is computed as (Tassoul et al. 1990):

$B=-\frac{1}{\chi_{\mathrm{T}}} \sum_{1}^{M-1} \chi_{\mathrm{X}_{\mathrm{i}}} \frac{\mathrm{d} \ln X_{i}}{\mathrm{~d} \ln P}$

where:

$\chi_{\mathrm{X}_{\mathrm{i}}}=\left(\frac{\mathrm{d} \ln P}{\mathrm{~d} \ln X_{i}}\right)_{\rho, \mathrm{T},\left\{\mathrm{X}_{\mathrm{j} \neq \mathrm{i}}\right\}}$.

The expression in Eq. (1) is rigorously valid for chemically homogeneous stars. In this equation (see also Eq. (2)), the dependence on the Brunt-Väisälä frequency is such that the asymptotic period spacing is larger when the mass and/or temperature of the model is lower. This trend is clearly evidenced by Fig. 2 for $M_{*} \gtrsim 0.25 M_{\odot}$, in which we depict the evolution of the asymptotic period spacing for all the sequences considered in this work. The higher values of $\Delta \Pi_{\ell}^{\mathrm{a}}$ for lower $M_{*}$ comes from the dependence $N \propto g$, where $g$ is the local gravity $\left(g \propto M_{*} / R_{*}^{2}\right)$. On the other hand, the higher values of $\Delta \Pi_{\ell}^{\mathrm{a}}$ for lower $T_{\text {eff }}$ result from the dependence $N \propto \sqrt{\chi}_{T}$, with $\chi_{T} \rightarrow 0$ for increasing degeneracy $(T \rightarrow 0)$.

Note that, however, the increase of $\Delta \Pi_{\rho}^{\mathrm{a}}$ with $T_{\text {eff }}$ is not monotonous for the sequences with $M_{*} \lesssim 0.25 M_{\odot}$. In fact, for this range of masses, the period spacing experiences a local minimum before resuming the final growth trend with decreasing effective temperature according to the predictions of Eq. (2). That minimum in $\Delta \Pi_{\ell}^{\mathrm{a}}$ is found at lower $T_{\text {eff }}$ as we go to lower masses. This somewhat weird behavior is induced by the evolving shape of the $\mathrm{He} / \mathrm{H}$ chemical interface. We postpone an explanation for this feature of $\Delta \Pi_{\ell}^{\mathrm{a}}$ to Sect. 3.5.

The strong dependence of the period spacing on $M_{*}$ as evidenced by Fig. 2 could be used, in principle, to infer the stellar mass of pulsating low-mass white dwarfs, provided that enough consecutive pulsation periods were available from observations. Such a prospect could be severely complicated by the fact that the period spacing of pulsating white dwarfs also depends on the thickness of the outer envelope (Tassoul et al. 1990), where $\Delta \Pi_{\ell}^{\mathrm{a}}$ is larger for thinner envelopes. This is particularly true in the context of low-mass He-core white dwarfs, in which the models of ELM objects harbor $\mathrm{H}$ envelopes that are several times thicker

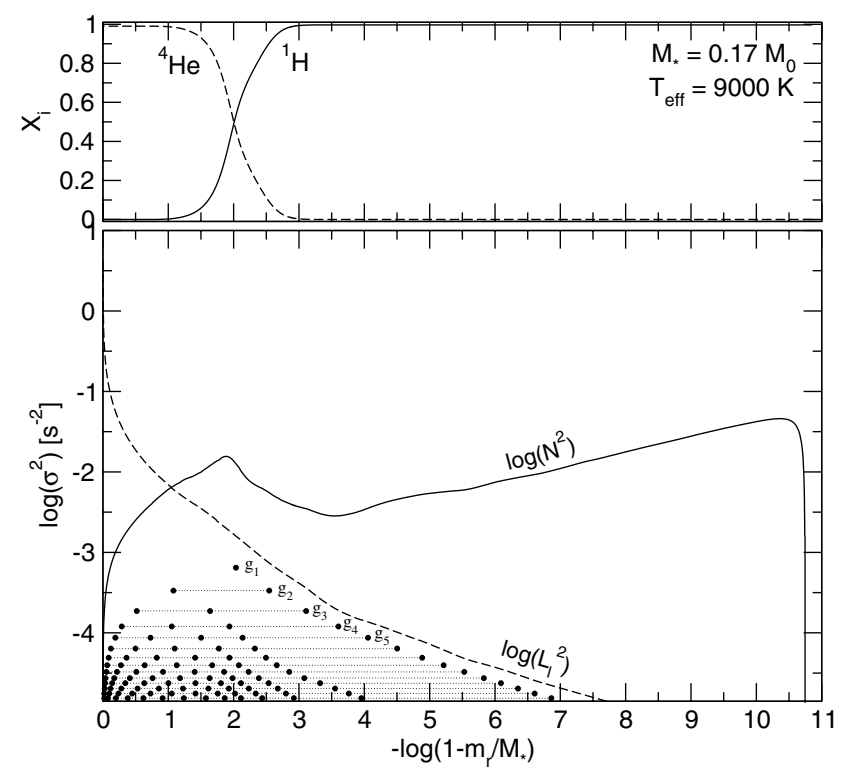

Fig. 3. The internal chemical profiles of $\mathrm{He}$ and $\mathrm{H}$ (upper panel) and the propagation diagram - the run of the logarithm of the squared critical frequencies - (lower panel) corresponding to an ELM white dwarf model of $M_{*}=0.17 M_{\odot}$ and $T_{\text {eff }} \approx 9000 \mathrm{~K}$. Dots connected with thin dotted lines correspond to the spatial location of the nodes of the radial eigenfunction of low-order dipole $(\ell=1) g$-modes.

than more massive models. Then, regarding the value of $\Delta \Pi_{\ell}^{\mathrm{a}}$, and for a fixed $T_{\text {eff }}$, a model with a low mass and a thick $\mathrm{H}$ envelope could readily mimic a more massive model with a thinner envelope. We envisage that, if a rich spectrum of observed periods were available, this ambiguity could be broken by including additional information of the mode trapping properties, which yield clues about the H envelope thickness (see Sect. 3.3).

\subsection{Chemical profiles, critical frequencies and eigenfunctions}

Our low-mass white dwarf models are made of a He core surrounded by a $\mathrm{H}$ outer envelope. In between, there is a smooth transition region shaped by the action of microscopic diffusion, which is self consistently accounted for in LPCODE. In the upper panels of Figs. 3 and 4 we display the internal chemical profiles for $\mathrm{He}$ and $\mathrm{H}$ corresponding to two template models of $M_{*}=0.17 M_{\odot}$ and $M_{*}=0.45 M_{\odot}$, respectively, at $T_{\text {eff }} \approx 9000 \mathrm{~K}$. The less massive model is representative of ELM white dwarfs, and the more massive model is representative of massive He-core white dwarfs $\left(0.20 \lesssim M_{*} / M_{\odot} \lesssim 0.45\right)$. It is worth emphasizing two important differences between these template models. First, the ELM model has a $\mathrm{H}$ envelope that is $\approx 35$ times thicker than the massive model. As mentioned in the Introduction, this is the result of the very different evolutionary history of the progenitor stars. Second, the other notorious difference between the chemical structures shown in Figs. 3 and 4 is the width of the $\mathrm{He} / \mathrm{H}$ transition region. Specifically, this interface is markedly wider for the ELM model than for the massive one; see Sect. 3.5 for an explanation.

The features in the chemical profiles leave strong signatures in the run of the squared critical frequencies, in particular in $N^{2}$, clearly shown in the lower panels of Figs. 3 and 4, which depict the propagation diagrams (Cox 1980; Unno et al. $1989)$ corresponding to these models. The squared Lamb frequency is defined as $L_{\ell}^{2}=\ell(\ell+1) c_{\mathrm{s}}^{2} / r^{2}$, where $c_{\mathrm{s}}$ is the local 


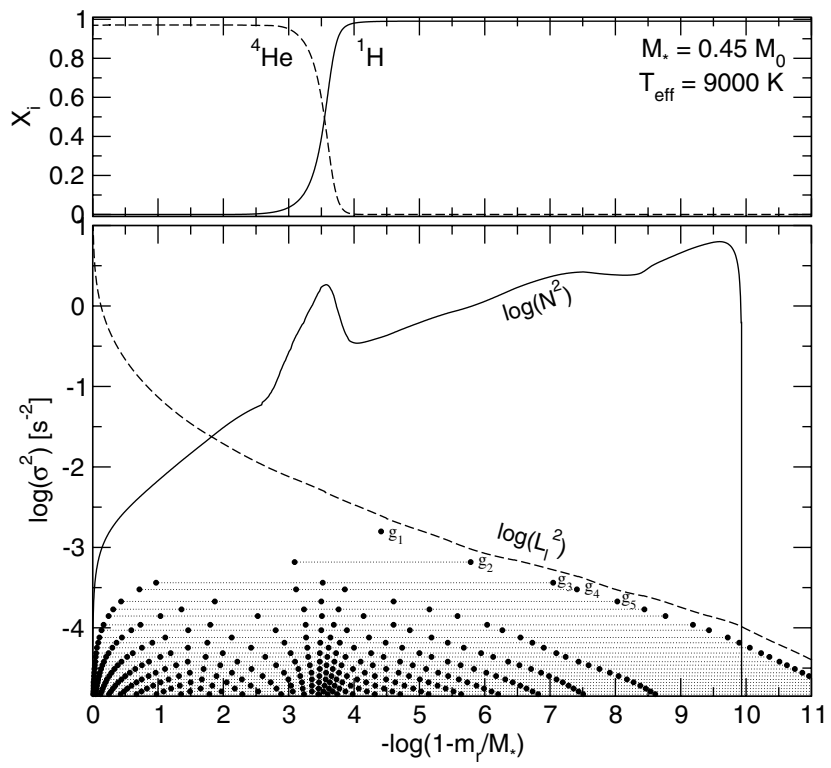

Fig. 4. Same as in Fig. 3, but for a $M_{*}=0.45 M_{\odot}$ white dwarf model.

adiabatic sound speed. $g$-modes propagate in the regions where $\sigma^{2}<N^{2}, L_{\ell}^{2}$, where $\sigma$ is the oscillation frequency. Note the very different shape of $N^{2}$ for both models. To begin with, the squared Brunt-Väisälä frequency for the ELM model is globally lower than for the case of the $0.45 M_{\odot}$ model. This has to do with the lower gravity that characterizes the former model, which translates into smaller values of $N^{2}$. Ultimately, a lower Brunt-Väisälä frequency profile produces a pulsation spectrum with longer pulsation periods.

Another different feature between the propagation diagrams of both models is related to the bump at the $\mathrm{He} / \mathrm{H}$ transition region. This is notoriously more narrow and pronounced for the massive model than for the ELM one. As can be seen in Fig. 4, for the $0.45 M_{\odot}$ model this feature visibly affects the distribution of the nodes of the radial eigenfunctions, which cluster at the precise location of the peak in $N^{2}$. In contrast, the distribution of nodes (and so, the shape of the eigenfunctions) is largely unaffected by the chemical interface in the case of the ELM model (Fig. 3). As we shall see in Sect. 3.3, this results in a weaker mode trapping as compared with the more massive models.

Finally, another important characteristic of the ELM model is that its squared Brunt-Väisälä frequency exhibits a local maximum at $-\log (q) \approx 2$, which coincides with the loci of the bump due to the $\mathrm{He} / \mathrm{H}$ transition region. But from there, $N^{2}$ increases very slightly outwards, without reaching much larger values in the surface layers. This is in contrast with the case of the $0.45 M_{\odot}$ model, in which the squared Brunt-Väisälä frequency exhibits larger values at the outer layers, resembling the situation in the $\mathrm{C} / \mathrm{O}$ core $\mathrm{DAV}$ white dwarf models (see Fig. 3 of Romero et al. 2012). This quite different shape of the run of $N^{2}$ has strong consequences on the propagation properties of eigenmodes. Specifically, and due to the particular shape of $N^{2}$ (that is, larger in the core than in the envelope; see also Fig. 2 of SEA10), the resonant cavity of the eigenmodes for the ELM model is circumscribed to the core regions $(-\log q \lesssim 2)$, whereas for the $0.45 M_{\odot}$, the propagation region extends along the whole model.

This can be appreciated in more detail in Figs. 5 and 6 , in which we plot the eigenfunctions $y_{1}=\xi_{r} / r$ and $y_{2}=$ $\left(\sigma^{2} / g\right) \xi_{h}$ (where $\xi_{r}$ and $\xi_{h}$ are the radial and the horizontal

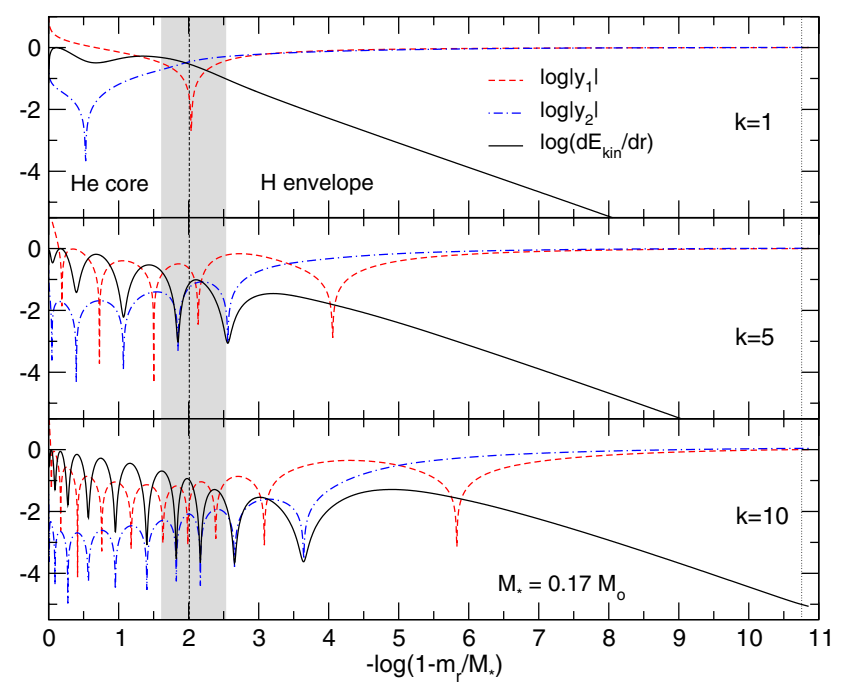

Fig. 5. The run of the logarithm of the absolute value of the eigenfunctions $y_{1}$ and $y_{2}$, and the density of kinetic energy $\mathrm{d} E_{\text {ekin }} / \mathrm{d} r$ for $\ell=1$ modes with $k=1$ (upper panel), $k=5$ (middle panel), and $k=10$ (lower panel), corresponding to the template model with $M_{*}=0.17 M_{\odot}$. The vertical dashed line and the associated shaded strip mark the location of the $\mathrm{He} / \mathrm{H}$ chemical transition region, whereas the vertical dotted line indicates the bottom of the outer convective zone.

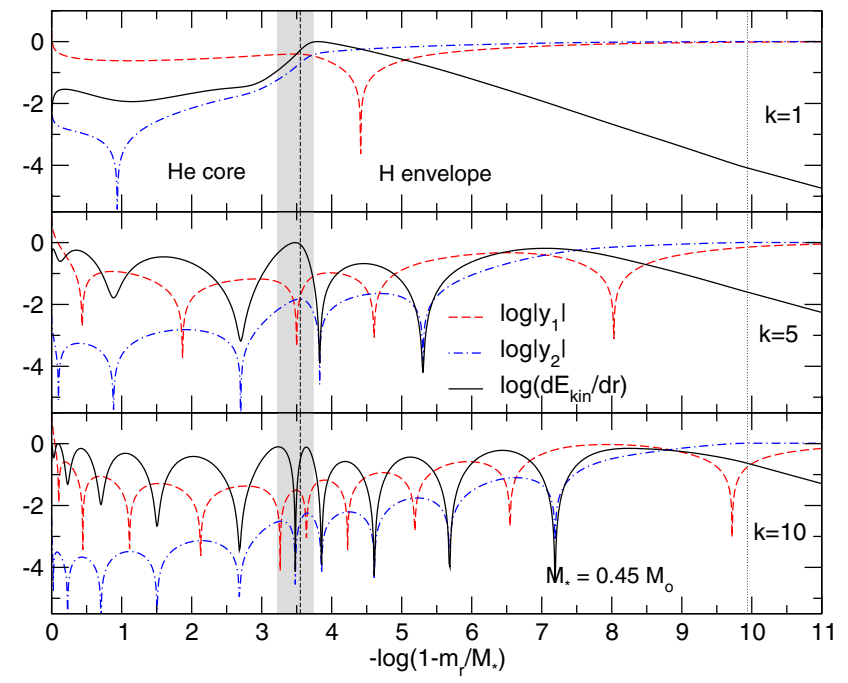

Fig. 6. Same as Fig. 5, but for the model with $M_{*}=0.45 M_{\odot}$.

displacements, respectively) and the density of oscillation kinetic energy $\mathrm{d} E_{\mathrm{kin}} / \mathrm{d} r$ (see Appendix A of Córsico \& Althaus 2006 for its definition) corresponding to modes with radial order $k=1,5$ and 10 for the two template models $\left(T_{\text {eff }} \approx 9000 \mathrm{~K}\right)$. From Fig. 5 it is apparent that most of the spatial oscillations of the modes in the ELM white dwarf model are restricted to the regions below the $\mathrm{He} / \mathrm{H}$ interface, i.e., most of nodes are located in the region with $-\log (q) \lesssim 2$. This plot should be compared with Fig. 2 of SEA10. In summary, $g$-modes in ELM white dwarfs probe mainly the stellar core ${ }^{2}$ and so, they have an enormous asteroseismic potential, as it was first recognized by SEA10.

In contrast, $g$-modes in more massive models (Fig. 6) oscillate all along the stellar structure, and not just at the core, although they are more selectively affected by mode-trapping

2 However, higher-order $g$-modes in the ELM model are less concentrated in the core than low-order ones, and probe a significant portion of the star. 


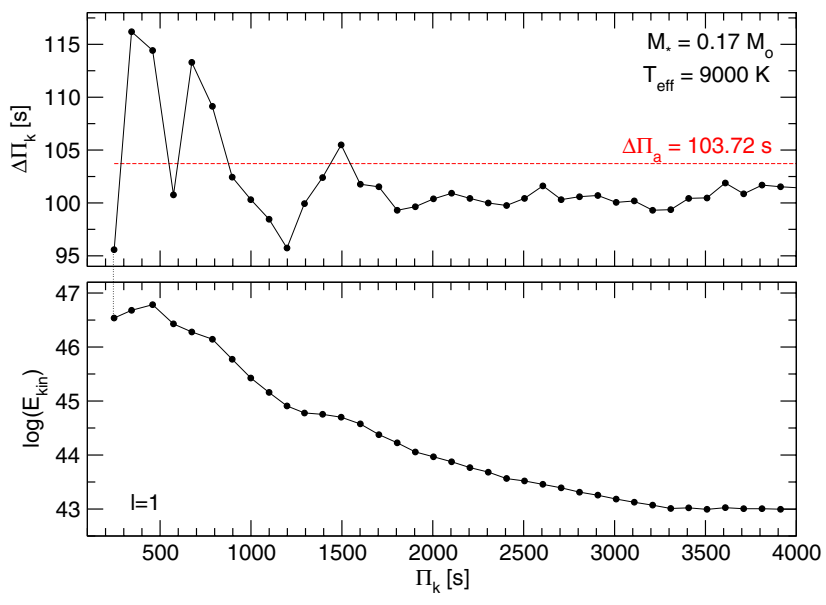

Fig. 7. The forward period spacing (upper panel) and the oscillation kinetic energy (lower panel) in terms of the $(\ell=1)$ periods for the model with $M_{*}=0.17 M_{\odot}$ and $T_{\text {eff }}=9000 \mathrm{~K}$. The red horizontal line in the upper panel corresponds to the asymptotic period spacing computed with Eq. (1).

effects produced by the compositional gradient at the $\mathrm{He} / \mathrm{H}$ interface. In this sense, low-mass He-core white dwarfs with $M_{*} \gtrsim$ $0.20 M_{\odot}$ behave qualitatively similar to their massive cousins, the $\mathrm{C} / \mathrm{O}$-core DAV white dwarf stars.

\subsection{Mode trapping}

The period spectrum of chemically homogeneous stellar models is characterized by a constant period separation, given very accurately by Eq. (1). However, current evolutionary calculations and different pieces of observational evidence indicate that white dwarf stars have composition gradients in their interiors (Althaus et al. 2010). The presence of one or more narrow regions in which the abundances of nuclear species (and so, the average molecular weight $\mu$ ) are spatially varying modifies the character of the resonant cavity in which modes should propagate as standing waves. Specifically, chemical interfaces act like reflecting boundaries that partially trap certain modes, forcing them to oscillate with larger amplitudes in specific regions and with smaller amplitudes outside of those regions. The requirement for a mode to be trapped is that the wavelength of its radial eigenfunction matches the spatial separation between two interfaces or between one interface and the stellar center or surface. This mechanical resonance, known as mode trapping, has been the subject of intense study in the context of stratified DA and DB white dwarf pulsations (Brassard et al. 1992; Bradley et al. 1993; Córsico et al. 2002a). In the field of PG 1159 stars, mode trapping has been extensively explored by Kawaler \& Bradley (1994) and Córsico \& Althaus (2006).

There are two clear signatures of mode trapping. One of them affects the distribution of the oscillation kinetic energy $\left(E_{\mathrm{kin}}\right)$ values in terms of the radial order (or the periods) of the modes. Since $E_{\text {kin }}$ is proportional to the integral of the squared amplitude of eigenfunctions weighted by the density (see Appendix A of Córsico \& Althaus 2006), modes propagating in the high-density environment typical of the deep interior of the star will exhibit higher values than modes that are oscillating in the low-density, external regions. Thus, a local maximum in $E_{\text {kin }}$ is usually associated to a mode partially confined to the core regions, and a local minimum corresponds generally to a mode partially trapped in the envelope.

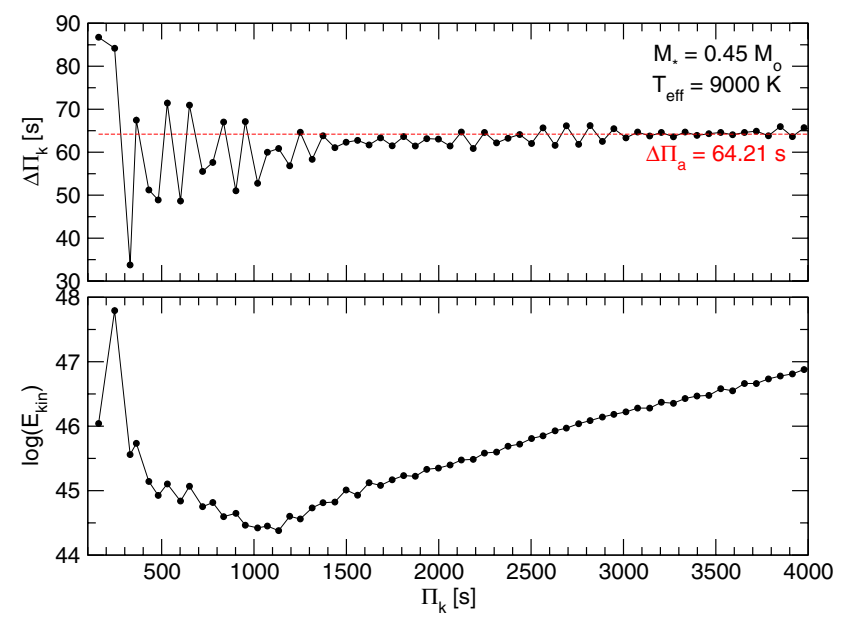

Fig. 8. Same as Fig. 7, but for the model with $M_{*}=0.45 M_{\odot}$.

The second, and more important from an observational point of view, signature of mode trapping is that the forward period spacing $\Delta \Pi_{k}\left(\equiv \Pi_{k+1}-\Pi_{k}\right)$, when plotted in terms of the pulsation period $\Pi_{k}$, exhibits strong departures from uniformity. It is the period difference between an observed mode and adjacent modes $(\Delta k= \pm 1)$ that matters as an observational diagnostic of mode trapping, at variance with $E_{\text {kin }}$, whose value is very difficult to estimate only from observations. For stellar models characterized by a single chemical interface, like the ones we are considering here, local minima in $\Delta \Pi_{k}$ usually correspond to modes trapped in the outer layers, whereas local maxima in $\Delta \Pi_{k}$ are associated to modes trapped in the core region.

In the upper panels of Figs. 7 and 8 we show the forward period spacing for the template models with $M_{*}=0.17 M_{\odot}$ and $M_{*}=0.45 M_{\odot}$, respectively. Lower panels depict the distribution of kinetic energy for the same models. The period spacings for the $0.17 M_{\odot}$ model reach the asymptotic value of $103.72 \mathrm{~s}$ for periods longer $(\approx 5500 \mathrm{~s})$ than the longest period shown in the plot. This is at variance with what happens in the case of the $0.45 M_{\odot}$ model, for which $\Delta \Pi_{k}$ approaches to the asymptotic value of $64.21 \mathrm{~s}$ at periods $\approx 1800 \mathrm{~s}$. The signatures of mode trapping are much more notorious in the case of the $0.45 M_{\odot}$ model than for the $0.17 M_{\odot}$ one. Indeed, mode trapping in the ELM template model is appreciable only in the $\Delta \Pi_{k}$ values for $\Pi_{k} \lesssim 1500 \mathrm{~s}$, and the $E_{\text {kin }}$ distribution is quite smooth for the complete range of periods shown. The weakness of mode-trapping in this model was anticipated in the previous section by virtue of the very wide and smooth $\mathrm{He} / \mathrm{H}$ chemical transition region. In contrast, for the $0.45 M_{\odot}$ model the trapping signatures are stronger in both the $\Delta \Pi_{k}$ and $E_{\text {kin }}$ distributions, although the kinetic energy exhibits a quite smooth distribution for periods longer than $\approx 2000 \mathrm{~s}$.

The fact that the asymptotic period spacing of the ELM model is not very rapidly reached (in terms of the pulsation periods) is not in contradiction with the weakness of mode trapping effects characterizing this model. This is because, even for chemically homogeneous models - which completely lack of mode trapping effects due to the absence of chemical gradients - the asymptotic regime is reached for modes with radial orders higher than a given limit (minimum) value of $k$. This is clearly shown in Fig. 6 of Córsico \& Benvenuto (2002), which displays the situation for a chemically homogeneous white dwarf model made of pure He.

In summary, our results indicate that mode trapping by the $\mathrm{He} / \mathrm{H}$ interface is important in low-mass He-core white dwarfs with masses $M_{*} \gtrsim 0.20 M_{\odot}$, but it is not an important issue in 


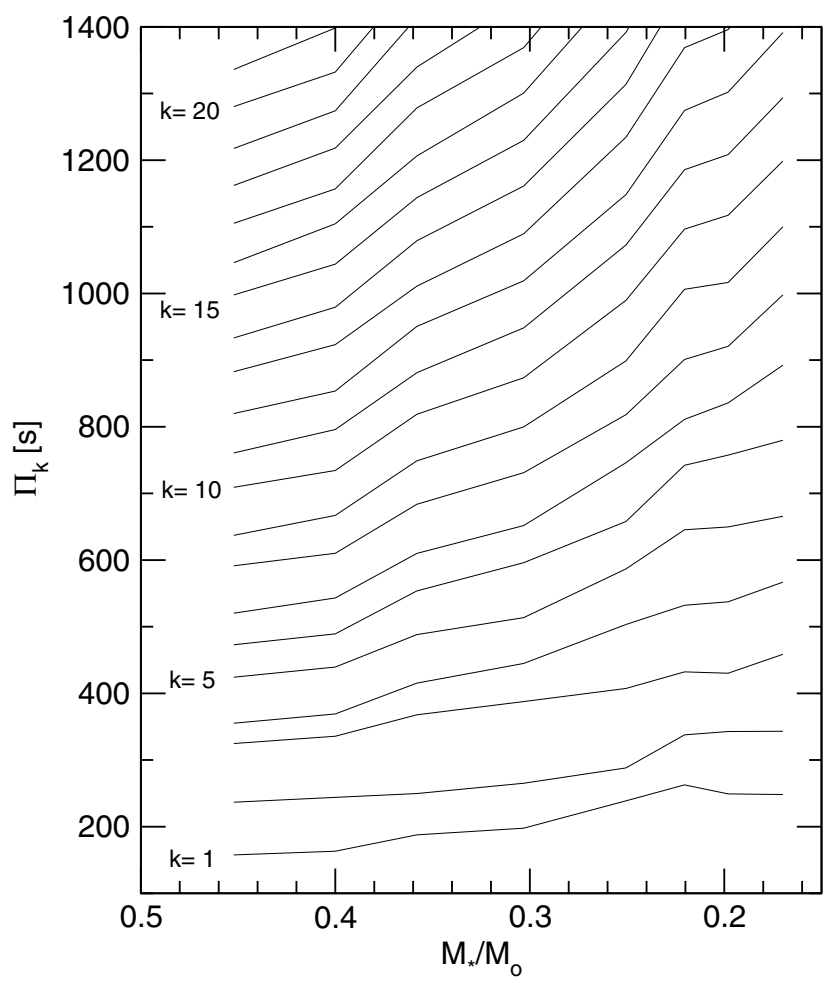

Fig. 9. Pulsation periods of $\ell=1 \mathrm{~g}$-modes in terms of the stellar mass for $T_{\text {eff }}=9500 \mathrm{~K}$. Periods increase with decreasing $M_{*}$.

ELM white dwarfs. This feature severely limits the seismological potential of these objects to constrain the thickness of the $\mathrm{H}$ envelope.

\subsection{Dependence of the pulsations with $M_{*}$ and $T_{\text {eff }}$}

As discussed in Sect. 3.1, the period spacing and the periods vary as the inverse of the Brunt-Väisälä frequency. This critical frequency, in turn, increases with larger stellar mass and with higher effective temperature. As a result, the pulsation periods are longer for smaller mass (lower gravity) and lower effective temperature (increasing degeneracy). The influence of the stellar mass on the pulsation periods is shown in Fig. 9, where we plot $\Pi_{k}$ for $\ell=1$ in terms of $M_{*}$ for a fixed value of $T_{\text {eff }}$. Note that the increase of $\Pi_{k}$ with decreasing $M_{*}$ is less pronounced for low-order modes than for higher-order ones. This is in agreement with the behavior typical of the ZZ Ceti star models (see Bradley 1996). In Fig. 10 we show the evolution of the pulsation periods with $T_{\text {eff }}$ for the case of models with $M_{*}=0.30 M_{\odot}$. The lengthening of the periods as the effective temperature drops is evident, although the effect is less pronounced than the decrease with the stellar mass (as compared with Fig. 9).

The effect of varying $M_{*}$ on the period-spacing and kineticenergy distributions for a fixed $T_{\text {eff }}$ is displayed in Fig. 11, in which we present the results for three white dwarf models with stellar masses of $M_{*}=0.17,0.30$ and $0.45 M_{\odot}$ and the same effective temperature $\left(T_{\text {eff }} \approx 9000 \mathrm{~K}\right)$. As expected, $\Delta \Pi_{k}$ strongly changes with $M_{*}$, as $\Delta \Pi^{\mathrm{a}}$ does. In fact, the average of the $\Delta \Pi_{k}$ values varies from $\approx 60 \mathrm{~s}$ to $\approx 100 \mathrm{~s}(\approx 60 \%)$ when the stellar mass changes from $0.45 M_{\odot}$ to $0.17 M_{\odot}$. Also, substantial changes in the kinetic energy distribution are expected when we vary the stellar mass, as evidenced by the lower panel of the Fig. 11.

Finally, we examine the effect of changing $T_{\text {eff }}$ on $\Delta \Pi_{k}$ and $E_{\text {kin }}$ with fixed $M_{*}$ in Fig. 12, where we show these quantities in

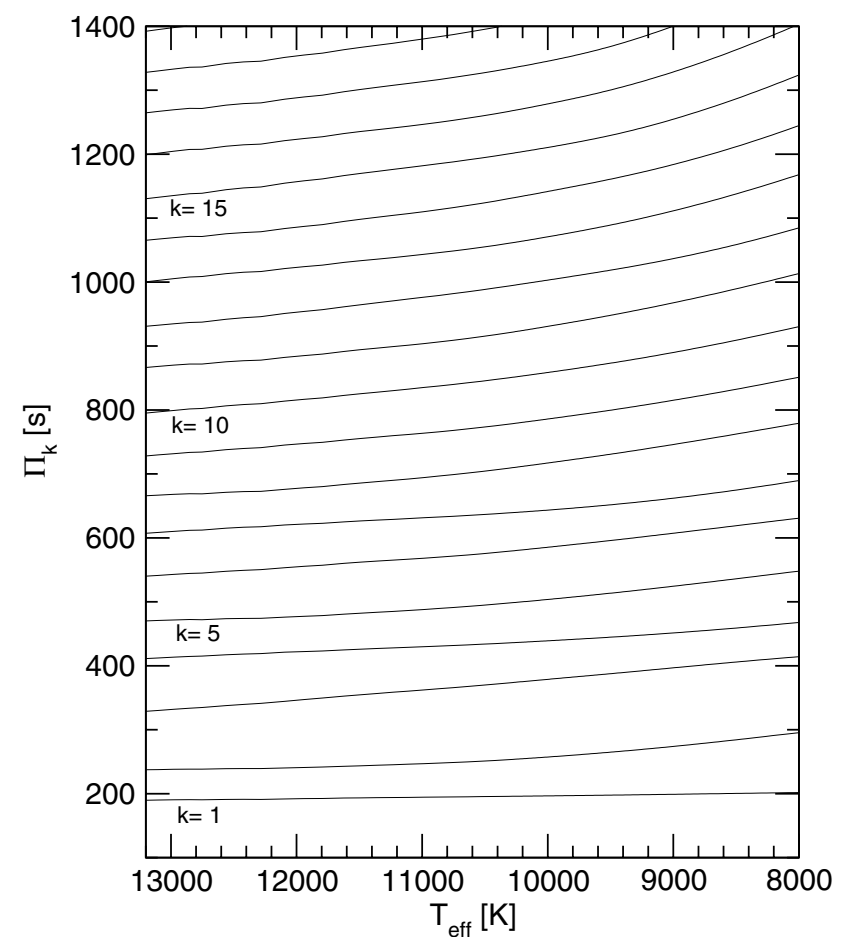

Fig. 10. Pulsation periods of $\ell=1 g$-modes in terms of the effective temperature for $M_{*}=0.30 M_{\odot}$. Periods increase with decreasing $T_{\text {eff }}$.

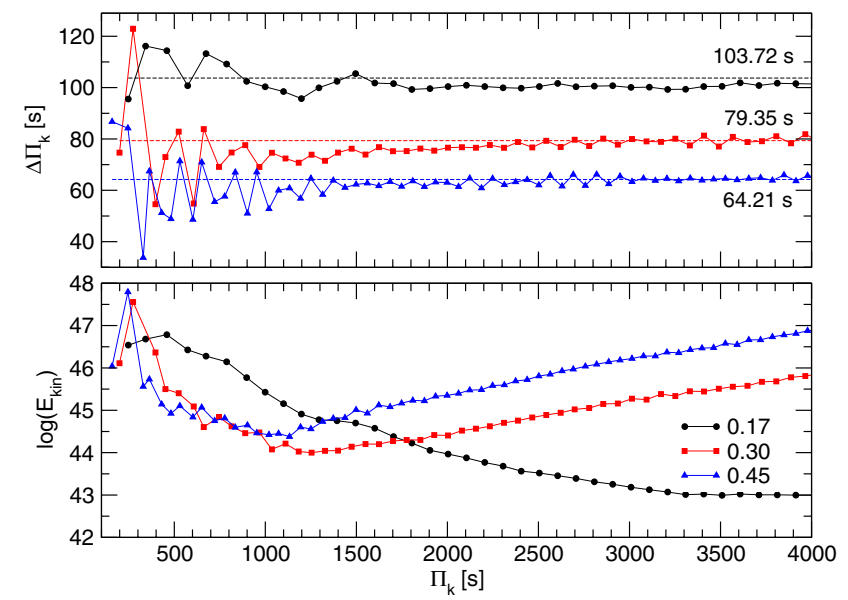

Fig. 11. The dipole forward period spacing (upper panel) and the oscillation kinetic energy (lower panel) in terms of the periods for models with $M_{*}=0.17 M_{\odot}, M_{*}=0.30 M_{\odot}$, and $M_{*}=0.45 M_{\odot}$, all of them characterized by a $T_{\text {eff }}=9000 \mathrm{~K}$. The horizontal lines in the upper panel correspond to the asymptotic period spacings computed with Eq. (1).

terms of the pulsation periods for a model with $M_{*}=0.30 M_{\odot}$ and three different values of the effective temperature. The sensitivity of the period spacing and the kinetic energy on $T_{\text {eff }}$ are negligible for short periods ( $\Pi_{k} \lesssim 500 \mathrm{~s}$ ) but it is quite appreciable for longer periods. Specifically, the average of the $\Delta \Pi_{k}$ values varies from $\approx 70 \mathrm{~s}$ to $\approx 85 \mathrm{~s}(\approx 20 \%)$ when the effective temperature changes from $12000 \mathrm{~K}$ to $8000 \mathrm{~K}$. Thus, the dependence of $\Delta \Pi_{k}$ with $T_{\text {eff }}$ is substantially weaker than with $M_{*}$. The kinetic energy values also are quite sensitive to changes in $T_{\text {eff }}$, at least for periods longer than $\approx 500 \mathrm{~s}$. For the hottest model depicted in Fig. $12\left(T_{\text {eff }}=12000 \mathrm{~K}\right)$, the more energetic modes are those with $k=1, \ldots, 5$, because they oscillate deep in the star where the density is high. However, for the cooler models $(9500$ and $8000 \mathrm{~K}$ ), the kinetic energy of the high-order modes, which 


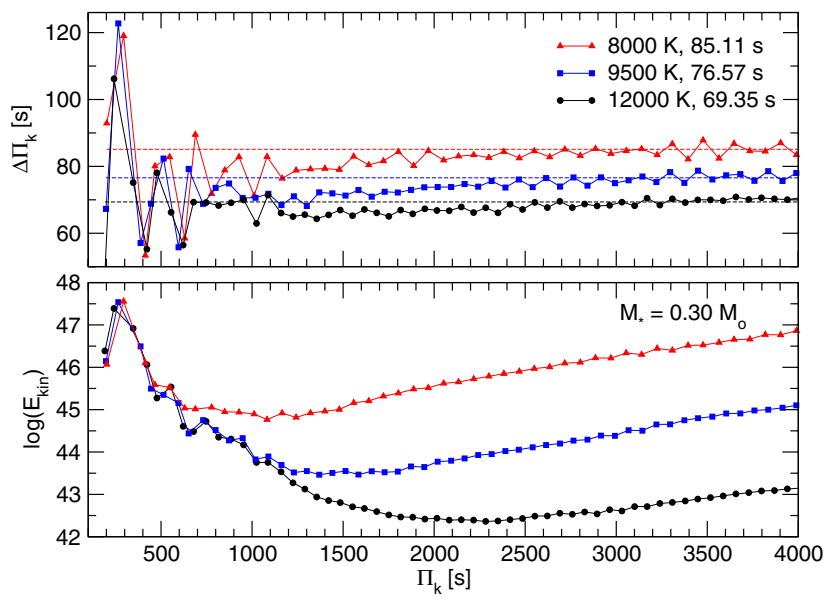

Fig. 12. Same as Fig. 11, but for the case of a model with $M_{*}=0.30 M_{\odot}$ and three different effective temperatures of $T_{\text {eff }}=12000,9500$ and $8000 \mathrm{~K}$.

are characterized by eigenfunctions mainly concentrated in the outer layers, strongly increase. This is because the decrease in $T_{\text {eff }}$ produces a deepening of the outer convection zone, and so, the high-order modes feel gradually the presence of convection as the white dwarf cools down. Since $g$-modes in a convection zone become evanescent, such modes are forced to gain larger amplitudes at regions somewhat below the base of the outer convection zone, where the density is larger. Since $E_{\text {kin }}$ is proportional to the integral of the squared eigenfunctions, weighted by $\rho$, these modes oscillate with larger energies. However, loworder modes remain rather insensitive to the thickening of the outer convection zone.

\subsection{Effects of element diffusion}

Finally, we discuss the effects that evolving chemical profiles have on the pulsation properties of low-mass, He-core white dwarfs. The effect of element diffusion on DAV stars has been explored by Córsico et al. (2002b). Time-dependent element diffusion, which is fully taken into account in our computations, strongly modifies the shape of the $\mathrm{He}$ and $\mathrm{H}$ chemical profiles as the white dwarf cools, causing $\mathrm{H}$ to float to the surface and He to sink down. In particular, diffusion not only modifies the chemical composition of the outer layers, but also the shape of the $\mathrm{He} / \mathrm{H}$ chemical transition region itself. This is clearly borne out from Fig. 13 for the case of the $0.17 M_{\odot}$ sequence in the $T_{\text {eff }}$ interval $(11000-8000 \mathrm{~K})$. For the model at $T_{\text {eff }}=11000 \mathrm{~K}$, the $\mathrm{H}$ profile is characterized by a diffusion-modeled doublelayered chemical structure, which consists in a pure $\mathrm{H}$ envelope atop an intermediate remnant shell rich in $\mathrm{H}$ and $\mathrm{He}$ (upper panel). This structure still remains, although to a much less extent, in the model at $T_{\text {eff }}=9500 \mathrm{~K}$ (middle panel). Finally, at $T_{\text {eff }}=8000 \mathrm{~K}$, the $\mathrm{H}$ profile has a single-layered chemical structure (lower panel). Element diffusion processes affect all the sequences considered in this paper, although the transition from a double-layered structure to a single-layered one occurs at different effective temperatures. The markedly lower surface gravity that characterizes the less massive model, results in a less impact of gravitational settling, and eventually in a wider chemical transition in the model with $M_{*}=0.17 M_{\odot}$. Because of this fact, the sequences with larger masses reach the single-layered structure at higher effective temperatures.

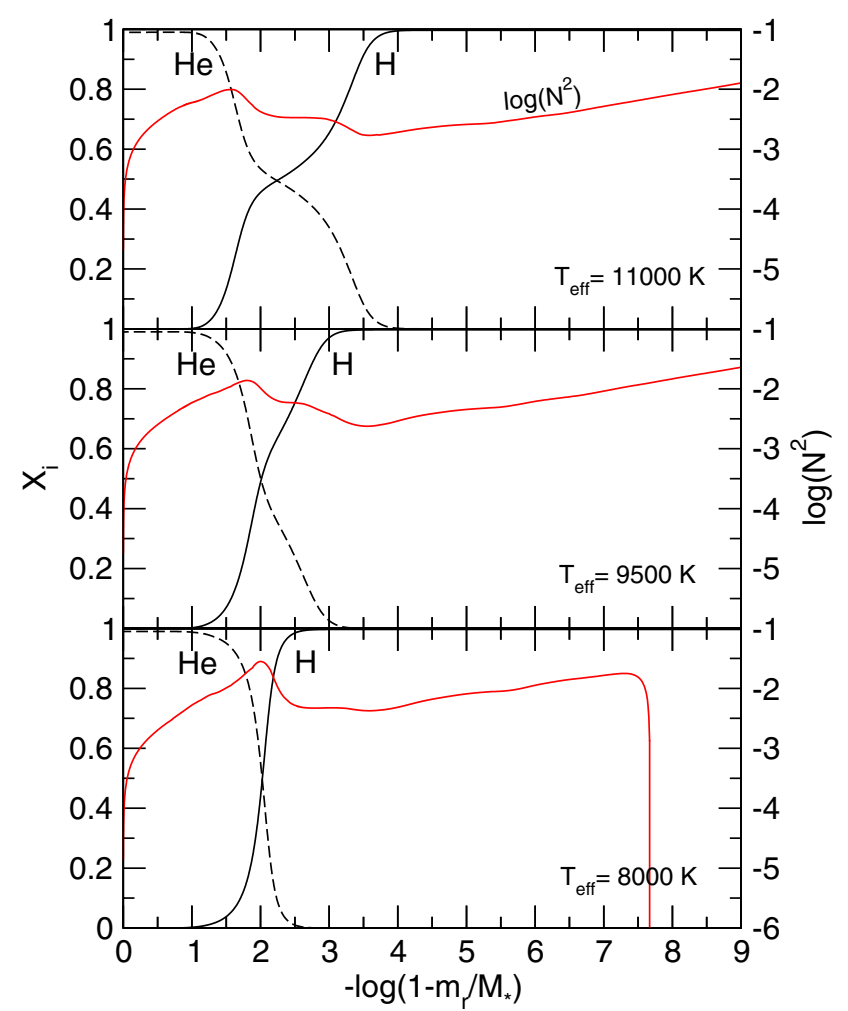

Fig. 13. The internal chemical profile of $\mathrm{H}$ and $\mathrm{He}$, and the logarithm of the squared Brunt-Väisälä frequency, for a model with $M_{*}=0.17 M_{\odot}$ at different effective temperatures, which are indicated in each panel.

The changes in the shape of the $\mathrm{He} / \mathrm{H}$ interface are translated into non-negligible changes in the profile of the Brunt-Väisälä frequency, as can be appreciated in the plot. In fact, at high effective temperatures, $N^{2}$ is characterized by two bumps, which merge into a single one when the chemical profile at the $\mathrm{He} / \mathrm{H}$ interface adopts a single-layered structure. The fact that the chemical profiles evolve with time in our models is at variance with the claim by SEA10, who argue that in their ELM models, diffusive equilibrium is valid in the $\mathrm{He} / \mathrm{H}$ transition region. In the light of our results, we conclude that this assumption is wrong.

In order to quantify the impact of the diffusively evolving chemical profiles on the period spectrum of low-mass He-core white dwarfs, we focus on the same ELM sequence analyzed in Fig. 13. Specifically, we have considered an additional sequence of models for which we switch off chemical diffusion in the LPCODE evolutionary code from $T_{\text {eff }} \approx 11000 \mathrm{~K}$ downwards. This allow us to isolate the effect that diffusion processes induce on the pulsation periods from those changes induced by cooling. We found substantial changes in the value of the periods when diffusion is neglected, depending on the specific mode considered and the value of the effective temperature. For instance, for the $k=3$ mode $\left(\Pi_{3} \approx 460 \mathrm{~s}\right)$ at $T_{\mathrm{eff}} \approx 9000 \mathrm{~K}$, a variation of $5 \%$ in the value of the period is expected.

We conclude that time-dependent element diffusion does affect the pulsation spectrum of low-mass and ELM white dwarfs, and that it must be taken into account in future pulsational analysis of ELM white dwarfs.

We close this section with an explanation to the behavior of $\Delta \Pi_{\ell}^{\mathrm{a}}$ in terms of the effective temperature for the sequences with $M_{*} \leq 0.22 M_{\odot}$, shown in Fig. 2 (Sect. 3.1). As we have seen, element diffusion processes lead to the formation of a double-layered configuration for the $\mathrm{H}$ profile in our models. 
This configuration consists in two regions where the He and $\mathrm{H}$ abundances exhibit a steeped spatial variation, which translates into two local features in the Brunt-Väisälä frequency. As evolution proceeds, the outer interface shifts downwards, gradually getting closer to the internal interface. The merging of the two bumps in $N$ tends to increase the integrand of Eq. (2), thus producing a decrease in the asymptotic period spacing. Thus, for a given $T_{\text {eff }}, \Delta \Pi_{\ell}^{\mathrm{a}}$ reaches a local minimum (see Fig. 2). Later, cooling again dominates and then $\Delta \Pi_{\ell}^{\mathrm{a}}$ increases, as expected. We found that the effective temperature at which $\Delta \Pi_{\ell}^{\mathrm{a}}$ reaches the minimum is higher for larger stellar masses. Thus, this minimum is not seen in Fig. 2 for the sequences with $M_{*} \geq 0.25 M_{\odot}$ because in these cases that minimum lies out of the range of $T_{\text {eff }}$ considered.

\section{Nonadiabatic computations and the case of SDSS J184037.78+642312.3}

Having thoroughly described in the previous sections the $g$-mode adiabatic seismic properties of our low-mass He-core white dwarf models, in what follows we will focus our attention on the only (up to now) ELM white dwarf discovered to be a pulsating star, SDSS J184037.78+642312.3. This DA white dwarf, which is the coolest $\left(T_{\text {eff }}=9100 \pm 170\right)$ and the lowest-mass $\left(M_{*} \approx 0.17 M_{\odot}\right)$ known pulsating $(1200 \lesssim \Pi \lesssim 4500 \mathrm{~s})$ object of this kind, was discovered by Hermes et al. (2012) in the context of a systematic search for variable He-core white dwarfs among the large number of ELM white dwarfs discovered through the ELM Survey (Brown et al. 2010, 2012; Kilic et al. 2011, 2012). The discovery of SDSS J184037.78+642312.3 opens the possibility to use the tools of white dwarf asteroseismology to obtain valuable information about the internal structure of low-mass white dwarf stars.

Here, we do not attempt an asteroseismological fitting to this star, because the period data are still far from be definitive. Rather, we concentrate mainly on the question of whether our low-mass He-core white dwarf models are able to predict the pulsations exhibited by SDSS J184037.78+642312.3 at the right effective temperature, stellar mass and range of observed periods.

In order to investigate the plausibility of excitation of pulsations in our models, we performed a linear stability analysis on our complete set of evolutionary sequences. We found that a dense spectrum of $g$-modes are excited by the $\kappa-\gamma$-mechanism acting in the $\mathrm{H}$ partial ionization zone for all the masses considered, and that there exists a well-defined blue (hot) edge of instability of He-core white dwarfs, which is the low-mass analog to the blue edge of the ZZ Ceti instability strip. We also found numerous unstable $p$-modes; the corresponding blue edge is hotter than the $g$-mode blue edge and has a lower slope. In Fig. 14 we show a zoom of the Fig. 1 at the region where SDSS J184037.78+642312.3 is located. We have included the location of the theoretical dipole $(\ell=1)$ blue edges of the instability domain of low-mass white dwarfs. The $g$-mode blue edge computed in this work is marked with a blue solid line, and it is extended towards lower gravities with a dashed blue line. The $p$-mode blue edge is displayed with a thin dotted line. Interestingly, SDSS J184037.78+642312.3 is slightly cooler than the derived $g$-mode blue edge, and so, our computations does predict long-period pulsations in this star. It should be kept in mind, however, that this result could change if a MLT prescription with less convective efficiency were assumed. Our stability analysis relies on the frozen-convection approximation that is known to give satisfactory predictions for the

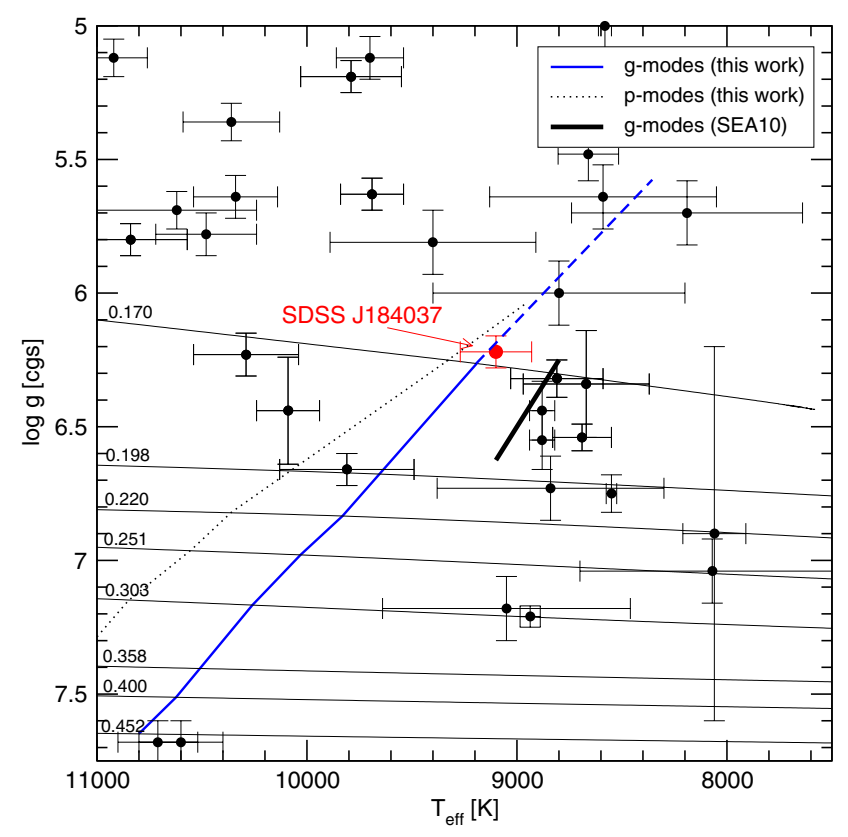

Fig. 14. A zoom of the $T_{\text {eff }}-\log g$ diagram shown in Fig. 1 showing the region where is located the only known (so far) pulsating ELM white dwarf, SDSS J184037.78+642312.3 (Hermes et al. 2012) displayed with a red circle. We include the approximate $\ell=1 \mathrm{~g}$-mode blue edge of the theoretical ELM instability domain derived by SEA10 (thick black solid line), and the $\ell=1 g$-mode blue edge computed in this work (blue solid line) for all the sequences considered, included that of $M_{*}=0.17 M_{\odot}$, and extended towards lower gravities (blue dashed line). We also show our $\ell=1 p$-mode blue edge of instability (thin dotted line).

location of the $g$-mode blue edge of other pulsating white dwarfs (DAVs, see Brassard \& Fontaine 1999; DBVs, see Córsico et al. 2009). Recently, a detailed study by van Grootel et al. (2012) showed that the blue edge of DAV models with $0.6 M_{\odot}$ computed with the frozen convection approximation does not dramatically differ from that obtained with a time-dependent convection treatment in the nonadiabatic computations. We note that also $p$-modes are predicted by our analysis to be destabilized in SDSS J184037.78+642312.3, although no short-period luminosity variations characteristic of acoustic modes have been detected in this star so far.

We emphasize that, besides SDSS J184037.78+642312.3, our nonadiabatic analysis predicts $g$ - and $p$-mode pulsational instabilities in the other stars at the right of the blue edges. We believe that it would be worthwhile to examine photometrically these stars in order to see whether they are pulsating or not.

The thick black solid line in Fig. 14 corresponds to the $g$-mode blue edge of the theoretical ELM white dwarf instability region derived by SEA10 by using the instability criterion $\Pi \leq$ $8 \pi \tau_{\text {th }}$ (Brickhill 1991; Wu \& Goldreich 1999) satisfied by $\ell=1$ and $k=1$ modes, where $\tau_{\text {th }}$ is the thermal timescale at the base of the convection zone. This criterion has been shown to be accurate in the case of ZZ Ceti pulsators, which are characterized by significantly higher gravities, but SEA10 were forced to extrapolate its predictions to the low-gravity regime of the ELM white dwarfs. The blue edge of SEA10 is about $350 \mathrm{~K}$ cooler than ours at $\log g \approx 6.2$, and in addition, its slope is somewhat larger, most likely the result of their using a different parametrization of convective efficiency ( $\alpha=1.0$, Steinfadt 2011). At variance with our results, the blue edge derived by SEA10 fails to predict pulsational instabilities in SDSS J184037.78+642312.3. We note 


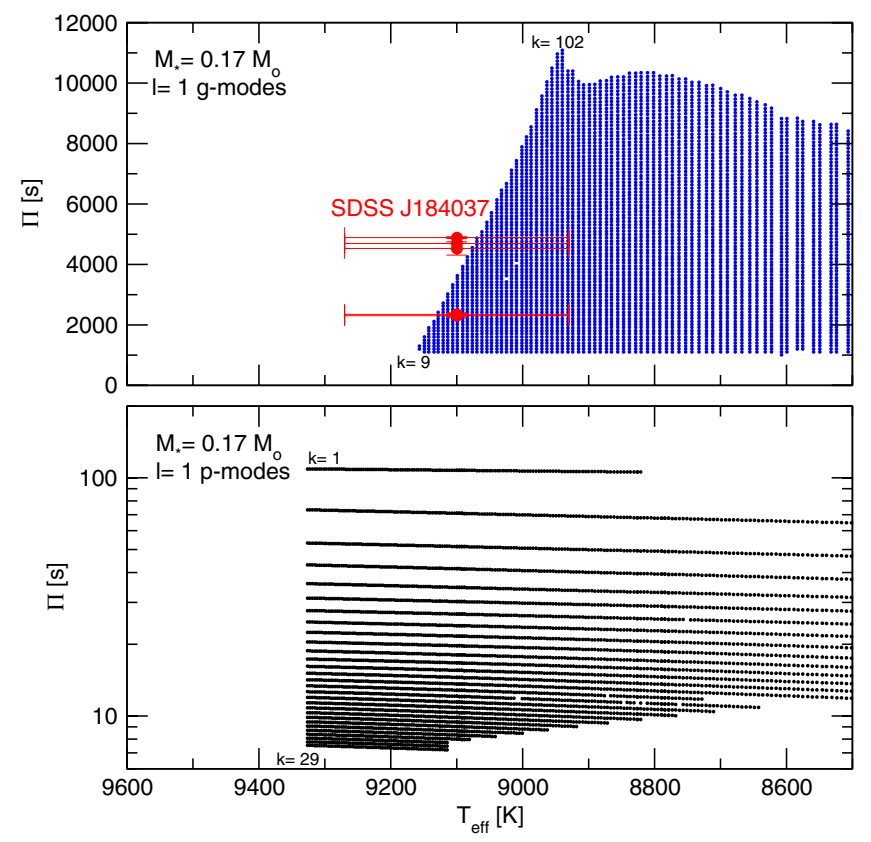

Fig. 15. Upper panel: the instability domain (blue dots) on the $T_{\mathrm{eff}}-$ $\Pi$ plane for $\ell=1 \mathrm{~g}$-modes corresponding to our set of models with $M_{*}=0.17 M_{\odot}$. Also shown are the periodicities measured in SDSS J184037.78+642312.3 (red points with error bars). Lower panel: same as upper panel, but for the $p$-mode instability domain (black dots).

that, in order to estimate the blue edge, SEA10 implicitly assume that the $k=1, \ell=1$ mode must be unstable, something that is not guaranteed at the outset without performing detailed nonadiabatic pulsation computations (see below). If the blue edge were derived by considering higher order modes $(k>1)$, then the discrepancy would be even larger because their blue edge should shift to lower effective temperatures.

We explored the domain of unstable dipole modes in terms of the effective temperature for the sequence of $M_{*}=0.17 M_{\odot}$. For this sequence, $g$-modes become unstable at $T_{\text {eff }} \approx 9200 \mathrm{~K}$. Figure 15 show the instability domains of both $g$ - and $p$-modes on the $T_{\text {eff }}-\Pi$ diagram. In the case of $g$-modes, we found that unstable modes have radial orders $k \geq 9(\Pi \gtrsim 1100 \mathrm{~s})$, where the most unstable modes for each value of $T_{\text {eff }}$ have the longest period. Modes with $k=6,7$ and 8 are only marginally unstable (their stability coefficients are extremely small) and have not been included in the plot. Finally, modes with $k=1, \cdots, 5$ are pulsationally stable. In the case of $p$-modes, we found that these modes destabilize at somewhat higher effective temperatures $\left(T_{\mathrm{eff}} \approx 9330 \mathrm{~K}\right)$. At variance with the case of $g$-modes, low-order $p$-modes with $k=1,2,3, \ldots, 29(109 \gtrsim \Pi \gtrsim 7.5 \mathrm{~s})$ simultaneously become unstable at the blue edge, being the most unstable ones (largest stability coefficients) those with the shortest periods. For the unstable models, the asymptotic frequency spacing of $p$-modes, defined as (Unno et al. 1989):

$\Delta v^{\mathrm{a}}=\left(2 \int_{0}^{R} \frac{\mathrm{d} r}{c_{\mathrm{S}}}\right)^{-1}$,

ranges from $\Delta v^{\mathrm{a}}=4.67 \mathrm{mHz}$ (at $T_{\mathrm{eff}} \approx 9330 \mathrm{~K}$ ) to $\Delta v^{\mathrm{a}}=$ $6.20 \mathrm{mHz}$ (at $T_{\text {eff }} \approx 8000 \mathrm{~K}$ ) for our $M_{*}=0.17 M_{\odot}$ sequence.

We have completed a reanalysis of the periods detected in SDSS J184037.78+642312.3 using nearly $33.0 \mathrm{~h}$ of photometric observation from October 2011 to July 2012. Our observing and reduction techniques are identical to those described in Hermes et al. (2012), but include more than $27.0 \mathrm{~h}$ of additional observations. Aliasing of the data has made determining the periods especially difficult, so we have made every attempt to be as conservative as possible in our error estimates. We include in Fig. 15 the periods detected in SDSS J184037.78+642312.3 as red dots with their corresponding uncertainties.

The highest-amplitude mode excited in the star occurs at $4697.8 \pm 4.3 \mathrm{~s}(51 \pm 5 \mathrm{mma})$; this periodicity appears in the $5.5 \mathrm{~h}$ of October 2011 discovery data, but was the secondhighest alias of the 4445 s mode quoted by Hermes et al. (2012). Additionally, we see evidence of variability at the following periods: $4310 \pm 200 \mathrm{~s}(28 \pm 3 \mathrm{mma}), 2309 \pm 60 \mathrm{~s}(25 \pm 3 \mathrm{mma})$, $2400 \pm 120 \mathrm{~s}(21 \pm 3 \mathrm{mma}), 4890 \pm 270 \mathrm{~s}(21 \pm 3 \mathrm{mma})$, and $2094 \pm 50 \mathrm{~s}(17 \pm 3 \mathrm{mma})$. These periodicities may be independently excited modes, but it is possible that the shorter-period modes are nonlinear combination frequencies of the longer modes. Additionally, this ELM white dwarf has an unseen companion; spectroscopic observations by Brown et al. (2012) find it to be in a $4.6 \mathrm{~h}$ binary with a minimum $0.64 M_{\odot}$ companion. If the ELM white dwarf is synchronized (or nearly synchronized) with the orbital period, it is possible that the modes just short and long of the $4697.8 \mathrm{~s}$ mode may be rotationally split components rather than independent modes.

Our $g$-mode stability computations are in good agreement with the range of periods observed in SDSS J184037.78+ 642312.3, as shown in Fig. 15. The small discrepancy for the longest periods can easily be accommodated taking into account the uncertainties in $T_{\text {eff }}$, which are known to be severe because the uncertainties characterizing the atmosphere models of very low-mass white dwarfs (Steinfadt et al. 2012).

Finally, we consider an ELM white dwarf model representative of SDSS J184037.78+642312.3. From our suite of models, we found a $0.17 M_{\odot}$ white dwarf model with $T_{\text {eff }}=9099 \mathrm{~K}$, $\log g=6.28$, and $\log \left(L / L_{\odot}\right)=0.0156$. The asymptotic period spacing of $g$-modes is $\Delta \Pi^{\mathrm{a}}=103.47 \mathrm{~s}$, and the asymptotic frequency spacing for $p$-modes is $\Delta v^{\mathrm{a}}=4.87 \mathrm{mHz}$. In Table 2 we show the $g$-mode periods, period spacings, rates of period change and oscillation kinetic energies corresponding to this model. Note that the longest observed period $(\approx 4700 \mathrm{~s})$ should correspond in our model to a $\ell=1 g$-mode with a very high radial order, $k \approx 44-45$. By examining the radial eigenfunction and density of kinetic energy of these modes, we found that most $(\approx 64 \%)$ of their nodes lay within the He core, although a significant number of nodes (16) are located at regions beyond the $\mathrm{He} / \mathrm{H}$ interface. In other words, the observed mode with period $\approx 4700$ s would sample a significant portion of the interior of the star, besides the stellar core. The $\ell=1$ asymptotic period spacing, of $\approx 103 \mathrm{~s}$ is more than two times longer than for a normal $0.6 M_{\odot} \mathrm{ZZ}$ Ceti star at $T_{\text {eff }} \approx 12000 \mathrm{~K}$. So, in addition to the low gravities and effective temperatures, the pulsational signatures of ELM white dwarfs should make them easy to distinguish them from the well-known, C/O-core ZZ Ceti stars.

\section{Summary and conclusions}

We have presented in this paper the first comprehensive theoretical study of the seismic properties of low-mass, He-core white dwarfs with masses in the range $0.17-0.46 M_{\odot}{ }^{3}$. We have employed fully evolutionary stellar structures representative of these stars, extracted from the sequences of low-mass He-core white dwarfs published by Althaus et al. (2009). These

3 Detailed tabulations of the the stellar models, chemical profiles, and pulsation periods of $g$ - and $p$-modes for different stellar masses and effective temperatures are available at the CDS and at our web site http: //www . fcaglp . unlp . edu . ar/evolgroup. 
Table 2. $g$-mode pulsation quantities of the ELM white dwarf model representative of the pulsating star SDSS J184037.78+642312.3, characterized by $M_{*}=0.17 M_{\odot}, T_{\text {eff }}=9099 \mathrm{~K}, \log g=6.28$, and $\log \left(L / L_{\odot}\right)=0.0156$.

\begin{tabular}{ccccc}
\hline \hline$k$ & $\Pi_{k}$ & $\Delta \Pi_{k}$ & $\mathrm{~d} \Pi_{k} / \mathrm{d} t$ & $\log E_{\text {kin }}$ \\
& {$[\mathrm{s}]$} & {$[\mathrm{s}]$} & {$\left[10^{-16} \mathrm{~s} / \mathrm{s}\right]$} & {$[\mathrm{erg}]$} \\
\hline 1 & 249.50 & 93.29 & 1.88 & 46.49 \\
2 & 342.79 & 116.73 & 0.16 & 46.68 \\
3 & 459.52 & 112.23 & 3.54 & 46.75 \\
4 & 571.75 & 100.36 & 10.23 & 46.39 \\
5 & 672.10 & 114.66 & 11.66 & 46.30 \\
6 & 786.77 & 109.52 & 11.13 & 46.17 \\
7 & 896.29 & 103.81 & 6.09 & 45.80 \\
8 & 1000.11 & 100.69 & 4.97 & 45.45 \\
9 & 1100.79 & 98.68 & 4.85 & 45.15 \\
10 & 1199.47 & 96.00 & 5.10 & 44.88 \\
11 & 1295.48 & 98.84 & 6.07 & 44.72 \\
12 & 1394.31 & 102.87 & 8.31 & 44.67 \\
13 & 1497.19 & 104.76 & 10.17 & 44.62 \\
14 & 1601.95 & 103.29 & 9.71 & 44.48 \\
15 & 1705.23 & 102.36 & 9.08 & 44.31 \\
16 & 1807.60 & 99.646 & 7.41 & 44.10 \\
17 & 1907.24 & 100.88 & 9.49 & 43.95 \\
18 & 2008.12 & 100.55 & 6.64 & 43.80 \\
19 & 2108.68 & 101.61 & 8.15 & 43.70 \\
20 & 2210.29 & 101.45 & 7.43 & 43.57 \\
21 & 2311.74 & 101.16 & 9.14 & 43.47 \\
22 & 2412.90 & 100.94 & 6.54 & 43.34 \\
23 & 2513.84 & 100.89 & 7.67 & 43.25 \\
24 & 2614.74 & 101.62 & 6.15 & 43.15 \\
25 & 2716.36 & 102.42 & 9.29 & 43.08 \\
26 & 2818.77 & 101.40 & 9.21 & 42.98 \\
27 & 2920.18 & 102.07 & 7.63 & 42.89 \\
28 & 3022.25 & 100.59 & 7.40 & 42.79 \\
29 & 3122.84 & 102.15 & 7.17 & 42.70 \\
30 & 3224.99 & 100.49 & 7.64 & 42.62 \\
31 & 3325.48 & 100.94 & 6.18 & 42.53 \\
32 & 3426.42 & 100.50 & 5.37 & 42.47 \\
33 & 3526.92 & 102.39 & 5.32 & 42.44 \\
34 & 3629.32 & 102.39 & 10.02 & 42.42 \\
35 & 3731.71 & 103.03 & 7.97 & 42.38 \\
36 & 3834.75 & 102.31 & 8.05 & 42.34 \\
37 & 3937.05 & 103.63 & 9.80 & 42.30 \\
38 & 4040.69 & 102.87 & 11.09 & 42.27 \\
39 & 4143.56 & 102.58 & 8.82 & 42.21 \\
40 & 4246.14 & 102.66 & 11.93 & 42.18 \\
41 & 4348.80 & 102.79 & 9.66 & 42.13 \\
42 & 4451.59 & 102.89 & 14.30 & 42.12 \\
43 & 4554.47 & 103.09 & 13.62 & 42.10 \\
44 & 4657.56 & 103.53 & 16.79 & 42.10 \\
45 & 4761.09 & 103.67 & 16.37 & 42.09 \\
46 & 4864.77 & 103.99 & 22.30 & 42.11 \\
47 & 4968.76 & 105.33 & 21.29 & 42.11 \\
\hline & & & & \\
\hline
\end{tabular}

models were derived by considering the evolutionary history of progenitor stars with supersolar metallicities, and accounting for a time-dependent treatment of the gravitational settling and chemical diffusion, as well as of residual nuclear burning. We have explored the adiabatic pulsation properties of these models, including the expected range of periods and period spacings, the propagation properties and mode trapping of pulsations, the regions of period formation, as well as the dependence on the effective temperature and stellar mass. For the first time, we assessed the pulsation properties of He-core white dwarfs with masses in the range $0.20-0.45 M_{\odot}$. In particular, we emphasize the expected differences in the seismic properties of objects with $M_{*} \gtrsim 0.20 M_{\odot}$ and the ELM white dwarfs $\left(M_{*} \lesssim 0.20 M_{\odot}\right)$. The pulsation properties of ELM white dwarfs have been explored recently by SEA10. Our work can be considered as complementary to that study. We have also explored the role of time-dependent element diffusion in ELM white dwarf models on their pulsational properties. Finally, we have computed $g$ - and $p$-mode blue edges of the instability domain for these stars through a nonadiabatic stability analysis. In particular, we attempted to determine if our evolutionary/pulsation models are able to predict the pulsations exhibited by SDSS J184037.78+642312.3 both in the right effective temperature and mass, and also in the correct range of periodicities.

We summarize our findings below.

- Extremely low mass (ELM) white dwarfs $\left(M_{*} \lesssim 0.20 M_{\odot}\right)$ have a $\mathrm{H}$ envelope that is much thicker than for massive He-core white dwarfs $\left(\approx 0.20-0.45 M_{\odot}\right)$, due to the very different evolutionary history of the progenitor stars. In particular, ELM white dwarfs did not experience diffusion-induced $\mathrm{CNO}$ flashes, thus they harbor thick $\mathrm{H}$ envelopes.

- By virtue of the thicker $\mathrm{H}$ envelope characterizing ELM white dwarfs, they experience $\mathrm{H}$ burning via the $\mathrm{pp}$ chain, and consequently their evolution is extremely slow. This feature makes these stars excellent candidates to become pulsating objects (SEA10). However, He-core white dwarfs with $M_{*} \approx 0.40-0.45 M_{\odot}$ should have evolutionary timescales of the same order, also making them attractive targets for current searches of variable low-mass white dwarfs.

- The thickness of the $\mathrm{He} / \mathrm{H}$ transition region is markedly wider for the ELM white dwarfs than for massive He-core white dwarfs. This is due to the markedly lower surface gravity characterizing ELM white dwarfs, that results in a less impact of gravitational settling, and eventually in a wider chemical transition.

- The Brunt-Väisälä frequency for ELM white dwarfs is globally lower than for the case of massive objects, due to the lower gravity characterizing ELM white dwarfs. A lower Brunt-Väisälä frequency profile leads to longer pulsation periods.

- The bump of $N^{2}$ at the $\mathrm{He} / \mathrm{H}$ transition region is notoriously more narrow and pronounced for the massive white dwarfs than for the ELM white dwarfs. This feature results in a weaker mode trapping in ELM white dwarfs, something that severely limits their seismological potential to constrain the thickness of the $\mathrm{H}$ envelope.

- As already noted by SEA10, the Brunt-Väisälä frequency of the ELM white dwarfs is larger in the core than in the envelope. This is in contrast with the case of models with $M \gtrsim 0.20 M_{\odot}$, in which the Brunt-Väisälä frequency exhibits larger values at the outer layers, thus resembling the situation encountered in ZZ Ceti stars. So, $g$-modes in ELM white dwarfs probe mainly the stellar core and have an enormous asteroseismic potential, as it was first recognized by SEA10.

- Similarly to ZZ Ceti stars, the $g$-mode asymptotic period spacing in low-mass He-core white dwarfs is sensitive primarily to the stellar mass, and to a somewhat less extent, to the effective temperature. Specifically, $\Delta \Pi_{\ell}^{\mathrm{a}}$ is longer for lower $M_{*}$ and $T_{\text {eff }}$. Also, there is a non-negligible dependence with the thickness of the $\mathrm{H}$ envelope, where $\Delta \Pi_{\ell}^{\mathrm{a}}$ is longer for thinner $\mathrm{H}$ envelopes (small $M_{\mathrm{H}}$ ). Typically, the asymptotic period spacing range from $\approx 55 \mathrm{~s}$ for $M_{*}=$ $0.45 M_{\odot}$ and $T_{\text {eff }}=11500 \mathrm{~K}$, up to $\approx 110 \mathrm{~s}$ for $M_{*}=$ $0.17 M_{\odot}$ and $T_{\text {eff }}=8000 \mathrm{~K}$ (see Fig. 2). The evolution of the 
asymptotic period spacing in terms of the effective temperature is markedly influenced by the evolving chemical profiles due to element diffusion.

- Similar to what happens with the asymptotic period spacing, the spectrum of $g$-mode pulsation periods themselves is very sensitive to the stellar mass, the effective temperature and the thickness of the $\mathrm{H}$ envelope. Again, the largest dependence is with the stellar mass.

- Time-dependent element diffusion does appreciably affect the $g$-mode pulsation spectrum of low-mass white dwarfs, in particular ELM white dwarfs. In this regard, it is expected that diffusion processes substantially modify the $\mathrm{He} / \mathrm{H}$ chemical interface, and so the resulting period spectrum, for models with stellar masses $M_{*} \lesssim 0.22 M_{\odot}$. We emphasize that time-dependent element diffusion should be taken into account in future pulsational analysis of these stars.

- We obtained for the first time the $g$-mode blue (hot) edge of the instability domain for He-core low-mass white dwarfs with $0.20 \lesssim M_{*} / M_{\odot} \lesssim 0.45$. On the other hand, our $g$-mode blue edge for ELM white dwarfs $\left(M_{*} \lesssim 0.20 M_{\odot}\right)$ is hotter than that found by SEA10.

- We also found a $p$-mode blue edge of instability of low-mass He-core white dwarfs. So, according to our analysis, several known low-mass white dwarfs with masses below $\approx 0.30 M_{\odot}$ should be pulsating stars showing short and long periods associated to $p$ - and $g$-modes, respectively (see Fig. 14).

- Our stability analysis successfully predicts the pulsations oberved in the only known variable low-mass white dwarf, SDSS J184037.78+642312.3 (Hermes et al. 2012), at the right effective temperature, stellar mass and range of pulsation periods. Our computations also predict the presence of short-period pulsations in this star that, however, have not been detected by now.

- We found a representative model of SDSS J184037.78+ 642312.3 , with parameters $M_{*}=0.17 M_{\odot}, T_{\text {eff }}=9099 \mathrm{~K}$, $\log g=6.28, \log \left(L / L_{\odot}\right)=0.0156, \Delta \Pi^{\mathrm{a}}=103.47 \mathrm{~s}$ and $\Delta v^{\mathrm{a}}=4.87 \mathrm{mHz}$. According to this model, the longest pulsation period exhibited by this star $(\approx 4700 \mathrm{~s})$ would be associated to a $\ell=1 \mathrm{~g}$-mode with a very high radial order, $k=44-45$, which probably samples a significant portion of the star, apart from the core.

The recent discovery of SDSS J184037.78+642312.3, the first pulsating low-mass white dwarf star, has opened a new opportunity to sound the interiors of these kind of stars through asteroseismology. In the next years a significant number of these pulsating objects will likely be uncovered through systematic photometric searches like the ones performed by Steinfadt et al. (2012) and Hermes et al. (2012). Needless to say, in order to accurately decode the information embedded in the pulsation spectrum of these stars, it will be necessary to have at hand a large suite of detailed evolutionary/pulsational models of low-mass white dwarfs. This paper is aimed at fulfilling this requirement.

Acknowledgements. Part of this work was supported by AGENCIA through the Programa de Modernización Tecnológica BID 1728/OC-AR, and by the PIP 112- 200801-00940 grant from CONICET. This research has made use of NASA Astrophysics Data System.

\section{References}

Alexander, D. R., \& Ferguson, J. W. 1994, ApJ, 437, 879

Althaus, L. G., Serenelli, A. M., \& Benvenuto O. G. 2001, MNRAS, 323, 471
Althaus, L. G., Córsico, A. H., Gautschy, A., et al. 2004, MNRAS, 347, 125 Althaus, L. G., Serenelli, A. M., Panei, J. A., et al. 2005, A\&A, 435, 631 Althaus, L. G., Panei, J. A., Romero, A. D., et al. 2009, A\&A, 502, 207

Althaus, L. G., Córsico, A. H., Isern, J., \& García-Berro, E. 2010, A\&ARv, 18 471

Bassa, C. G. 2006, Ph.D. Thesis, Universiteit Utrecht

Bassa, C. G., van Kerkwijk, M. H., \& Kulkarni, S. R. 2003, A\&A, 403, 1067

Beauchamp, A., Wesemael, F., Bergeron, P., et al. 1999, ApJ, 516, 887

Bradley, P. A. 1996, ApJ, 468, 350

Bradley, P. A., Winget, D. E., \& Wood, M. A. 1993, ApJ, 406, 661

Brassard, P., \& Fontaine, G. 1999, Stellar Structure: Theory and Test of Connective Energy Transport, 173, 329

Brassard, P., Fontaine, G., Wesemael, F., \& Hansen, C. J. 1992, ApJS, 80, 369

Brickhill, A. J. 1991, MNRAS, 251, 673

Brown, W. R., Kilic, M., Allende Prieto, C., \& Kenyon, S. J. 2010, ApJ, 723, 1072

Brown, W. R., Kilic, M., Allende Prieto, C., \& Kenyon, S. J. 2012, ApJ, 744, 142

Burgers, J. M. 1969, Flow Equations for Composite Gases (New York: Academic Press)

Cassisi, S. Pothekin, A. Y., Pietrinferni, A., Catelan, M., \& Salaris, M. 2007, ApJ, 661, 1094

Castanheira, B. G., \& Kepler, S. O. 2008, MNRAS, 385, 430

Castanheira, B. G., \& Kepler, S. O. 2009, MNRAS, 396, 1709

Córsico, A. H., \& Althaus, L. G. 2006, A\&A, 454, 863

Córsico, A. H., \& Benvenuto, O. G. 2002, ApSS, 279, 281

Córsico, A. H., Althaus, L. G., Benvenuto, O. G., \& Serenelli, A. M. 2002a, A\&A, 387, 531

Córsico, A. H., Benvenuto, O. G., Althaus, L. G., \& Serenelli, A. M. 2002b, MNRAS, 332, 392

Córsico, A. H., García-Berro, E., Althaus, L. G., \& Isern, J. 2004, A\&A, 427, 923

Córsico, A. H., Althaus, L. G., \& Miller Bertolami, M. M. 2006, A\&A, 458, 259

Córsico, A. H., Althaus, L. G., Miller Bertolami, M. M., \& García-Berro, E. 2009, J. Phys. Conf. Ser., 172, 012075

Cox, J. P. 1980, Theory of stellar pulsations (Princeton: Princeton University Press)

Driebe, T., Schönberner, D., Blöcker, T., \& Herwig, F. 1998, A\&A, 339, 123

Dziembowski, W. 1971, Acta Astron., 21, 289

Fontaine, G., \& Brassard, P. 2008, PASP, 120, 1043

García-Berro, E., Torres, S., Althaus, L. G., et al. 2010, Nature, 465, 194

Haft, M., Raffelt, G., \& Weiss, A. 1994, ApJ, 425, 222

Hansen, B. M. S. 2005, ApJ, 635, 522

Hermes, J. J., Montgomery, M. H., Winget, D. E., et al. 2012, ApJ, 750, L28

Iben, I. Jr., \& Tutukov, A. V. 1986, ApJ, 311, 742

Iglesias, C. A., \& Rogers, F. J. 1996, ApJ, 464, 943

Itoh, N., Hayashi, H., Nishikawa, A., \& Kohyama, Y. 1996, ApJS, 102, 41

Kalirai, J. S., Bergeron, P., Hansen, B. M. S., et al. 2007, ApJ, 671, 748

Kawaler, S. D., \& Bradley, P. A. 1994, ApJ, 427, 415

Kawka, A., \& Vennes, S. 2009, A\&A, 506, L25

Kepler, S. O., Kleinman, S. J., Nitta, A., et al. 2007, MNRAS, 375, 1315

Kilic, M., Brown, W. R., Allende Prieto, C., Pinsonneault, M. H., \& Kenyon, S. J. 2007, ApJ, 664, 1088

Kilic, M., Brown, W. R., Allende Prieto, C., et al. 2011, ApJ, 727, 3

Kilic, M., Brown, W. R., Allende Prieto, C., et al. 2012, ApJ, 751, 141

Kleinman, S. J., Kepler, S. O., Koester, D., et al. 2012, ApJ, submitted

Magni, G., \& Mazzitelli, I. 1979, A\&A, 72, 134

Marsh, T. R., Dhillon, V. S., \& Duck, S. R. 1995, MNRAS, 275, 828

Panei, J. A., Althaus, L. G., Chen, X., \& Han, Z. 2007, MNRAS, 382, 779

Romero, A. D. 2012, Ph.D. Thesis, University of Buenos Aires

Romero, A. D., Córsico, A. H., Althaus, L. G., et al. 2012, MNRAS, 420, 1462

Sarna, M., Ergma, E., \& Gerškevitš-Antipova, J. 2000, MNRAS, 316, 84

Siess, L. 2007, A\&A, 476, 893

Silvotti, R., Østensen, R. H., Bloemen, S., et al. 2012, MNRAS, in press

Steinfadt, J. D. 2011, Ph.D. Thesis, University of California

Steinfadt, J. D. R., Bildsten, L., \& Arras, P. 2010, ApJ, 718, 441 (SEA10)

Steinfadt, J. D. R., Bildsten, L., Kaplan, D. L., et al. 2012, PASP, 124, 1

Tassoul, M., Fontaine, G., \& Winget, D. E. 1990, ApJS, 72, 335

Tremblay, P.-E., Bergeron, P., \& Gianninas, A. 2011, ApJ, 730, 128

Unno, W., Osaki, Y., Ando, H., Saio, H., \& Shibahashi, H. 1989, Nonradial Oscillations of Stars (University of Tokyo Press), 2nd. edn.

van Grootel, V., Dupret, M.-A., Fontaine, G., et al. 2012, A\&A, 539, A87

van Kerkwijk, M. H., Bassa, C. G., Jacoby, B. A., \& Jonker, P. G. 2005, Binary Radio Pulsars, ASP, eds. F. A. Rasio, \& I. H. Stairs, 328, 357

Winget, D. E., \& Kepler, S. O. 2008, ARA\&A, 46, 157

Wu, Y., \& Godreich, P. 1999, ApJ, 519, 783 